\title{
Cyclic electron flow and ascorbate pathway play a role in survival of Chlamydomonas sp. UWO241 to long-term photooxidative stress
}

\author{
Sarah Stahl-Rommel ${ }^{1}$, Isha Kalra ${ }^{1}$, Susanna D’Silva ${ }^{1}$, Mark Hahn ${ }^{1}$, Marina Cvetkovska ${ }^{2}$, \\ and Rachael Morgan-Kiss ${ }^{1}$ \\ ${ }^{1}$ Miami University College of Arts and Science \\ ${ }^{2}$ University of Ottawa
}

August 17, 2020

\begin{abstract}
Under environmental stress plants and algae employ a variety of strategies to avoid damage to the photosynthetic apparatus and maintain photosynthetic capacity. To date, most studies on stress acclimation have focused on model organisms possessing limited tolerance to elevated stress levels. We compared the long-term acclimatory capacities of a mesophilic alga (Chlamydomonas raudensis SAG 49.72; SAG 49.72) and an Antarctic halotolerant psychrophile (Chlamydomonas sp. UWO 241; UWO 241) by monitoring photobiology, cyclic electron flow (CEF) and ROS defense in cultures acclimated to long-term low temperature, high salinity or high light stress. SAG 49.72 responded to long-term stress by increasing chlorophyll a/b ratio and redistributing absorbed light energy from photosystem II (PSII) to photosystem I (PSI). In contrast, the psychrophile exhibited faster half times for P700+ re-reduction under all treatments, suggesting high CEF rates. High CEF was accompanied by increased capacity for nonphotochemical quenching. Last, UWO 241 exhibited constitutively high activity of two key ascorbate cycle enzymes, ascorbate peroxidase and glutathione reductase, as well as a large ascorbate pool. Our results suggest that UWO 241 relies on high PSI-mediated CEF and ROS detoxification to protect the photosynthetic apparatus while minimizing energy expenditure on repairs.
\end{abstract}

\section{INTRODUCTION}

Photostasis is a phenomenon common to all photosynthetic organisms: it encompasses processes which contribute to balancing rates of photosynthetic energy absorbed with energy consumed by metabolism (Öquist \& Hüner, 2003). Disruption of photostasis is manifested as an accumulation of a reduced pool of the mobile electron acceptor, plastoquinone (PQ), leading to photooxidative stress. This phenomenon occurs under excessive light conditions; however, any environmental condition which impacts an organism's ability to use absorbed light energy can lead to an over-reduction of the PQ pool (Hüner et al., 2012; Morgan-Kiss, Priscu, Pocock, Gudynaite-Savitch, \& Hüner, 2006). Thus, any alteration in an organism's environment can exacerbate disruption to photostasis and enhance the probability of photooxidative stress, including day/night cycle, salinity, drought, heat, chilling, and nutrient status (Bartels \& Sunkar, 2005; Ensminger, Busch, \& Hüner, 2006; Liu, Qi, \& Li, 2012; Sharma, Jha, Dubey, \& Pessarakli, 2012; Takahashi \& Murata, 2008).

A major biproduct of unbalanced photosynthesis is the production of reactive oxygen species (ROS). ROS accumulates when the photosynthetic electron transport chain becomes over-reduced, causing oxidative injury and damage to proteins, lipids, nucleic acids, and many components of the photosynthetic apparatus (Apel \& Hirt, 2004; Asada, 1996; Møller, Jensen, \& Hansson, 2007; Sirikhachornkit \& Niyogi, 2010). Oxidative stress responses are distinct at the level of time scale and can be classified into mechanisms for short-term, acute oxidative stress occurring over seconds to minutes, or long-term, constitutive stress occurring over hours to years (Niyogi, 1999; Suzuki, Koussevitzky, Mittler, \& Miller, 2012). Short-term responses are nonheritable adjustments to physiology and biochemistry which avoid ROS production (Ledford, Chin, \& Niyogi, 
2007; Sirikhachornkit \& Niyogi, 2010). Common short-term stress response mechanisms are phototaxis, state transitions, nonphotochemical quenching (NPQ), and alternative electron transport pathways, such as the water-water cycle and PSI-associated CEF (Kozi Asada, 2000; Cournac et al., 2002; Minagawa, 2011; Müller, Li, \& Niyogi, 2001; Witman, 1993). Changes in gene expression and protein translation aid in maintenance of photostasis over longer time scales. Long-term responses can involve minimizing ROS production and/or increasing ROS detoxification, and include changes to antenna size or PSI/PSII stoichiometry increased $\mathrm{CO}_{2}$ fixation capacity, and activation of antioxidant pathways (Kozi Asada, 2006; Falk et al., 1994; Falk, Maxwell, Gray, Rezansoff, \& Hüner, 1993; Lucker \& Kramer, 2013; Tanaka \& Melis, 1997; Yamori, Makino, \& Shikanai, 2016). Enzymatic antioxidants used for ROS detoxification include superoxide dismutase (SOD), catalase (CAT), and enzymes of the ascorbate-glutathione (AsA-GSH) cycle (Noctor \& Foyer, 1998). Maintenance of high antioxidant capacity has been associated with tolerance to environmental stress in plants and algae (Aldesuquy, Baka, El-Shehaby, \& Ghanem, 2013; Chen, Zhang, \& Shen, 2011; Van Alstyne, Sutton, \& Gifford, 2020). The AsA-GSH pathway is particularly important for antioxidative defense in plants but appears to play a lesser role in algae and cyanobacteria (Foyer \& Halliwell, 1976; Foyer, Lopez-Delgado, Dat, \& Scott, 1997; Hu et al., 2008).

Some photosynthetic organisms have evolved to survive and grow under permanent stressful environments. Relative to the well-studied processes of short- and long-term stress acclimation, strategies of photosynthetic adaptation to permanent abiotic stress are significantly less understood. Low temperature environments are abundant at high latitudes (Young \& Schmidt, 2020): photopsychrophiles are photosynthetic organisms which are physiologically adapted to permanent low temperatures (Morgan-Kiss et al., 2006). The Antarctic Chlamydomonas sp. UWO 241 (UWO 241) was isolated from a permanently ice-covered, hypersaline lake and represents one of the few models for photosynthetic adaptation to combined low temperatures and high salinity (Cvetkovska, Hüner, \& Smith, 2017). Early studies reported that UWO 241 exhibits minimal capacity for short-term acclimatory mechanisms, such as the xanothophyll cycle and state transitions (Morgan-Kiss, Ivanov, \& Hüner, 2002; Morgan, Ivanov, Priscu, Maxwell, \& Hüner, 1998), and sensitivity to short-term thermal or high light stress (Morgan-Kiss, Ivanov, Williams, Mobashsher, \& Hüner, 2002; T. Pocock, Koziak, Rosso, Falk, \& Hüner, 2007). In lieu of short-term acclimation, UWO 241 has evolved to rely on constitutive mechanisms as a consequence of adaptation to permanent low temperatures and high salinity (Morgan-Kiss et al., 2006). While UWO 241 exhibits high susceptibility to high light stress, it also possesses the ability to rapidly recover from photoinhibition (Pocock et al., 2007). Despite the presence of cold-active thylakoid kinases, energy transfer from PSII to PSI uses a poorly understood spill-over mechanism (Szyszka-Mroz et al., 2019). In addition, under native low temperature and high salinity conditions, UWO 241 forms a novel PSI supercomplex which allows the organism to maintain a strong capacity for PSI-driven CEF (Cook et al., 2019; Szyszka-Mroz, Pittock, Ivanov, Lajoie, \& Hüner, 2015). The additional proton motive force (pmf) derived from CEF is used for constitutive capacity for NPQ and production of additional ATP in cells grown under high salinity (Kalra et al., 2020). The adjustments to the photosynthetic apparatus are accompanied by alterations in carbon metabolism, including upregulation of several enzymes within the Calvin Benson Bassham cycle (CBB), and key enzymes of the shikimate pathway, a high carbon flux pathway which synthesizes precursors for aromatic metabolites (Julkowska, 2020; Kalra et al., 2020). Together, these novel adaptive strategies allow UWO 241 to maintain robust growth and photosynthesis under the combined stress of permanent low temperature and high salinity.

While activation of CEF is known to be essential in plants and algae exposed to short-term stress, the discovery of a strong CEF capacity in a psychrophilic, halotolerant alga suggests that there is a previously unappreciated role for CEF during long-term adaptation to environmental stress. We hypothesized that UWO 241 utilizes CEF and ROS detoxification as long-term stress acclimation mechanisms to maintain photostasis and protect the photosynthetic apparatus from photooxidative damage. We tested this hypothesis by comparing growth physiology as well as PSII and PSI photochemistry in UWO 241 and a related mesophilic species, Chlamydomonas raudensis SAG 49.72, acclimated to long-term stress conditions (high light, low temperature, high salinity). We also monitored production of a major $\mathrm{ROS}\left(\mathrm{O}_{2}^{-}\right)$as well as activity of two key enzymes of the AsA-GSH pathway (Ascorbate Peroxidase, APX; Glutathione Reductase, GR). Our 
study shows that UWO 241 possesses robust ability to acclimate to long-term photooxidative stress by both avoiding ROS production by maintaining photostasis through CEF and relying on constitutive ROS detoxification. We suggest that this reliance on the redundant systems allow the organism to withstand long-term exposure to multiple stressors in its native habitat while minimizing energy expenditures for repair processes.

\section{MATERIALS AND METHODS}

\section{Strains, growth conditions and growth physiology}

Cultures of the psychrophilic Chlamydomonas sp. UWO 241 (CCMP1619) and a mesophilic strain, Chlamydomonas raudensis SAG 49.72, were grown in Bold's basal medium (BBM) (Nichols \& Bold, 1965) under ambient $\mathrm{CO}_{2}$ conditions in $250 \mathrm{~mL}$ Pyrex tubes submerged in temperature-regulated aquaria as described in Morgan-Kiss et al. (2008). The mesophilic SAG 49.72 was chosen for comparison as it has been used in several comparative studies with UWO 241 (Pocock, Vetterli, \& Falk, 2011; Szyszka-Mroz et al., 2019; Szyszka-Mroz et al., 2015; Szyszka, Ivanov, \& Hüner, 2007). Cultures were grown under either control (C) conditions or exposed to one of three long-term stress treatments: high light (HL), low temperature (LT) or high salt (HS). For control conditions, cultures were grown under temperature/light regimes of $8^{\circ} \mathrm{C} / 50$ $\mu \mathrm{mol}^{-2} \mathrm{~s}^{-1}$ and $20^{\circ} \mathrm{C} / 50 \mu \mathrm{mol}^{-2} \mathrm{~s}^{-1}$ for UWO 241 and SAG 49.72, respectively, and $\mathrm{NaCl}$ levels of $0.43 \mathrm{mM}$ for both strains (Table 1). Long-term stress conditions were chosen based on previous studies (Morgan-Kiss et al., 2006; Pocock et al., 2011; Szyszka et al., 2007) to reflect the maximum level of a particular stress to which the organism could fully acclimate, that is achieve exponential growth and high photochemical activity (maximum photosynthetic efficiency values, $\mathrm{F}_{\mathrm{V}} / \mathrm{F}_{\mathrm{M}}$, above 0.5). Long-term stress conditions for UWO 241 and SAG 49.72, respectively, were: i) high light, $250 \mu \mathrm{mol} \mathrm{m} \mathrm{m}^{-2} \mathrm{~s}^{-1}$ and $500 \mu \mathrm{mol} \mathrm{m} \mathrm{m}^{-2} \mathrm{~s}^{-1}$; ii) low temperature, $2^{\circ} \mathrm{C}$ and $11^{\circ} \mathrm{C}$; iii) high salinity, $700 \mathrm{mM} \mathrm{NaCl}$ and $100 \mathrm{mM} \mathrm{NaCl}$.

Growth kinetics were monitored as change in optical density at $750 \mathrm{~nm}$. All other measurements were performed on mid-log phase cultures. Chlorophyll a and b concentrations were determined from whole cell extractions in $90 \%$ acetone according to Jeffry \& Humphrey (1975).

\section{Room temperature chlorophyll fluorescence}

The activities of PSI and PSII were measured in dark-adapted exponentially growing cultures with a DualPAM-100 system (Heinz Walz GmbH, Effeltrich, Germany) as described in Szyska et al. (2007). All samples were supplemented with $10 \mathrm{mM}$ sodium biocarbonate and measurements were performed in a water-jacketed cuvette at the corresponding growth temperatures. The fluorescence parameters $\mathrm{F}_{\mathrm{V}} / \mathrm{F}_{\mathrm{M}}$ (maximum photochemical efficiency), qL (photochemical quenching or proportion of open PSII reaction centers), $\Phi$ (PSII) (quantum yield of photochemistry), and $\Phi$ (NPQ) (nonphotochemical energy dissipation from antenna quenching), and $\Phi(\mathrm{NO})$ (energy dissipation from non-regulated processes) were calculated during steady state photosynthesis at the growth temperature/irradiance/salinity regimes. Energy partition parameters of PSI were: quantum yield of PSI ( $\Phi$ PSI), acceptor side limitation ( $\Phi$ NA), and donor side limitation $(\Phi N D)$ (Schreiber \& Klughammer, 2008).

\section{Low temperature Chl a fluorescence $(77 \mathrm{~K})$}

Low temperature $(77 \mathrm{~K}) \mathrm{Chl}$ fluorescence emission spectra were measured using a Perkin Elmer Luminescence Spectrometer (LS50B) (Buckinghamshire, England) equipped with liquid nitrogen accessory. Algal cultures from dark-adapted (10 min) mid-log phase cultures were transferred to NMR tubes and flash frozen in liquid nitrogen. Fluorescence spectra were collected at the excitation wavelength of $435 \mathrm{~nm}$ and recorded at a slit width of $4 \mathrm{~nm}$ for excitation and emission. Decompositional analysis of fluorescence emission spectra in terms of five Gaussian bands was performed by a non-linear least squares algorithm according to Morgan-Kiss et al. (2002b) using the program OriginPro 8.5.1.

\section{P700 reduction/oxidation kinetics}

Far red or red light induced photooxidation of P700 was used to determine rates of CEF as described by Morgan-Kiss et al. (2002b). A volume of exponential phase cultures representing $25 \mu \mathrm{g}$ Chl a was dark 
adapted for $10 \mathrm{~min}$ and then filtered onto $25 \mathrm{~mm}$ GF/C filters (Whatman, Cat No. 1822-025). Filters were measured on the Dual-PAM 100 instrument using the leaf attachment. The proportion of photooxidizable P700 was determined by monitoring absorbance changes at $820 \mathrm{~nm}$ and expressed as the parameter $\left([?] \mathrm{A}_{820} / \mathrm{A}_{820}\right)$. The signal was balanced and the measuring light switched on. Far red (FR) light $(\lambda \max =715$ $\mathrm{nm}, 10 \mathrm{Wm}^{-2}$, Scott filter RG 715) was then switched on to oxidize P700. For the red light measurement, cells were pre-treated in the dark for $10 \mathrm{~min}$ in the presence of $10 \mu \mathrm{M}$ DCMU to inhibit electron transfer from PSII to PSI. After steady-state oxidation levels were reached, the FR or red light was switched off to re-reduce P700. The half time for the reduction of $\mathrm{P} 700^{+}$to P700 $\left(t_{\frac{1}{2}}\right.$ red $)$ was calculated as an estimate of relative rates of PSI-driven CEF (Ivanov, Morgan, Gray, Velitchkova, \& Hüner, 1998). The re-reduction time for P700 was calculated using the program OriginPro 8.5.1 using first order exponential decay kinetics.

\section{Ascorbate Pathway}

Glutathione reductase (GR) activity was measured using a glutathione reductase assay kit based on NADPH oxidations (Kit 703202, Cayman Chemicals, Ann Arbor). Mid-log phase cultures ( ${ }^{\sim} 3-10 \times 10^{6}$ cells) were collected by centrifugation and resuspended in GR assay buffer (50 mM potassium phosphate, $1 \mathrm{mM}$ EDTA, $\mathrm{pH} 7.5$ ). Cells were lysed by $4 \times 30 \mathrm{~s}$ beadbeating cycles. Twenty microliters of lysed cell supernatant was mixed with $100 \mu \mathrm{L}$ GR assay buffer and $20 \mu \mathrm{L}$ oxidized glutathione. The reactions were initiated with $50 \mu \mathrm{L}$ $\mathrm{NADPH}$, and oxidation of NADPH was measured kinetically over $10 \mathrm{~min}$ at $340 \mathrm{~nm}$ at an assay temperature of $25^{\circ} \mathrm{C}$. Activity was calculated by $\Delta \mathrm{A}_{340} \mathrm{~min}^{-1} \mathrm{mg}^{-1}$ protein using an NADPH extinction coefficient of $0.00373 \mu \mathrm{M}^{-1}$.

APX activity was measured according to (Venisse, Gullner, \& Brisset, 2001) with some modifications. Sample extracts were prepared as described for the GR activity assay. Ten microliters of supernatant was added to $190 \mu \mathrm{L}$ of reaction buffer ( $50 \mathrm{mM}$ potassium phosphate buffer, $\mathrm{pH} 7.8$ ), supplemented with $0.5 \mathrm{mM}$ ascorbic acid and $0.1 \mathrm{mM}$ hydrogen peroxide. Oxidation of ascorbate was monitored spectrophotometrically as a decrease $\mathrm{A}_{290}$ (extinction coefficient $0.00168 \mu \mathrm{M}^{-1}$ ) over 10 min to determine APX activity (Venisse et al., 2001).

For ascorbate quantitation, $25 \mathrm{~mL}$ of culture ( 6 to $7 \times 10^{7}$ total cells) was pelleted and washed once in HPLC grade $\mathrm{H}_{2} \mathrm{O}$. Pellets were resuspended in an extracted in $2 \mathrm{mM}$ ETA containing $5 \mathrm{mM}$ dithiothreitol and $1 \%$ orthophosphoric acid. Cells were broken using a bead beater $(2 \times 30$ s cycles $)$ and samples were centrifuged for $30 \mathrm{~min}$ at 19,000 $\mathrm{x} \mathrm{g}$ and total cellular ascorbate levels (ascorbate + dehydroascorbate) were determined using a commercial kit (Ascorbate Assay Kit, Cayman Chemical. \#700420).

The genome and transcriptome of UWO 241 were sequenced and assembled as described before (Cvetkovska et al., 2019). These datasets were screened for the presence of the genes encoding for enzymes of the AsAGSH cycle. Previously identified genes from the model alga Chlamydomonas reinhardtii were obtained from the Phytozome database (v12, Joint Genome Institute) and used as a query. Genomic sequences with a high degree of identity (E-value cutoff $10^{-20}$ ) were obtained and annotated using Geneious Prime (Biomatters Ltd, Auckland, New Zealand).

The amino acid sequence was predicted based on the gene coding sequence, and the identity of the enzyme was confirmed based on conserved motifs (Pitsch Witsch, \& Baier, 2010; Wu and Wang, 2019). Multiple sequence alignments were performed using Clustal Omega (Sievers and Higgins, 2018), and protein localization was predicted using PSORT (Horton et al., 2007) and PredAlgo (Tardif et al., 2012). Sequence data for the UWO241 genes can be found in GenBank/EMBL database under accession numbers listed in Table S2.

\section{Photoinhibition assays}

Short-term photoinhibitory and recovery treatments were performed on UWO 241 and SAG 49.72 cells grown under control growth conditions according to T. Pocock et al. (2007). Algal cells were harvested during mid-log phase of growth and placed under either high light (HL, $300 \mu \mathrm{mol} \mathrm{m} \mathrm{m}^{-2} \mathrm{~s}^{-1}$ ) or low temperature (LT, $\left.2^{\circ} \mathrm{C}\right)$ stress for $1 \mathrm{hr}$. For the HL treatment, cultures were maintained at their growth temperatures of $8^{\circ} \mathrm{C}$ (UWO 241) and $20^{\circ} \mathrm{C}$ (SAG 49.72). These conditions were chosen after testing sensitivity of both organisms 
to a range of light $\left(50,300\right.$ and $\left.500 \mu \mathrm{mol} \mathrm{m} \mathrm{m}^{-2} \mathrm{~s}^{-1}\right)$ and temperature $\left(2{ }^{\circ} \mathrm{C}, 8{ }^{\circ} \mathrm{C}\right.$ and $\left.20^{\circ} \mathrm{C}\right)$ treatments over three hours. Following the photoinhibitory treatment, cells were allowed to recover in the light $(20 \mu \mathrm{mol}$ $\mathrm{m}^{-2} \mathrm{~s}^{-1}$ ) for $2 \mathrm{hr}$. Photoinhibition and recovery was monitored at regular time intervals as the decline and recovery in $\mathrm{F}_{\mathrm{V}} / \mathrm{F}_{\mathrm{M}}$, respectively.

\section{Semi-quantitation of superoxide}

Superoxide $\left(\mathrm{O}_{2}^{-}\right)$levels were quantified according to (Förster, Osmond, \& Pogson, 2005) with some modifications. A volume representing $~ 12,500$ cells of UWO 241 or SAG 49.72 mid-log phase cultures grown under control conditions was treated with $20 \mu \mathrm{L}$ of $1 \mathrm{mM}$ nitroblue tetrazolium (NBT; Sigma) in the dark for 5 min prior to the stress treatment. Samples were then filtered onto $25 \mathrm{~mm}$ GF/C filters (Whatman, Cat No. 1822-025) and exposed to short-term stress either LT $\left(5^{\circ} \mathrm{C}\right)$ or HL $\left(300 \mu \mathrm{mol} \mathrm{m}{ }^{-2} \mathrm{~s}^{-1}\right)$ in an AlgaeTron 130 growth incubator (Photon Systems Instruments, Czech Republic) for up to $1 \mathrm{hr}$. Following treatment, filters were immediately immersed in $80 \%$ acetone to remove Chl, and then allowed to dry in a fume hood prior to imaging. $\mathrm{O}_{2}{ }^{-}$levels were measured semi-quantitatively by densitometric analyses using the program ImageJ (http://imagej.nih.gov/ij/).

\section{Statistical Analyses}

Statistical significance was determined using Student's paired t-test, uneven variance (OriginPro 8.5.1) between stress conditions and control within a single organism as well as between stress conditions in both organisms. Statistical significance was accepted when $\mathrm{P}$ value was less than 0.05 .

\section{RESULTS}

\section{Growth physiology and PSII photochemistry}

To compare the mechanisms by which the psychrophilic UWO 241 and the mesophilic SAG 49.72 acclimate to long-term environmental stress, the two strains were grown under control growth conditions and then shifted to one of three different stress conditions, representing high light (HL), low temperature (LT) and high salt (HS) (Table 1). First, it was confirmed that both strains could fully acclimate to each stress conditions by exhibiting log-phase growth and high photochemical activity in mid-log phase cultures. UWO 241 and SAG 49.72 acclimated to all long-term stress conditions as reflected in their ability to exhibit exponential growth and achieve high PSII photochemical efficiency $\left(\mathrm{F}_{\mathrm{V}} / \mathrm{F}_{\mathrm{M}}\right)$ under stress conditions (Figure S1; Table 2). Despite the fact that UWO 241 tolerates significantly lower temperature and high salinity levels compared with SAG 49.72, the two strains generally exhibited comparable growth rates under control vs. stress conditions, with the exception of high light treatment in SAG 49.72 which exhibited a 3 -fold increase in growth rate (Table 2). Chl a/b ratios were consistently lower in UWO 241 vs. SAG 49.72 regardless of growth conditions (Table 2). Moreover, SAG 49.72 cultures grown under all long-term stress conditions exhibited higher $\mathrm{Chl}$ a/b ratios compared with control cultures; although, this difference was only significant between the control and low temperature-grown cultures (Table 2).

Both organisms exhibited $\mathrm{qL}$ values $>0.70$ under control and all stress conditions, with the exception of HL-cultures which exhibited a reduction in qL of 1.2- and 4.7-fold relative to controls of UWO 241 and SAG 49.72, respectively. In addition, UWO 241 exhibited a significantly lower qL and FPSII relative to SAG 49.72 under control, low temperature and high salt (Table 2; Figure 1a,b). Both strains exhibited a decline in FPSII in response to HL which was accompanied by an increase in FNO and FNPQ in HL-SAG 49.72 and HL-UWO 241, respectively (Figure 1a,b). Last, we measured the capacity for NPQ in both strains grown under control versus stress conditions by measuring NPQ levels under a range of measuring irradiance levels. The mesophilic strain maintained low NPQ levels over the range of irradiance levels in cells acclimated to either control or stress conditions (Figure 2). In contrast, control UWO 241 cells exhibited a significantly higher NPQ capacity compared with that of SAG 49.72. Acclimation to all stress conditions further enhanced the NPQ capacity of UWO 241 by 1.4 to 2.8 -fold, with HL- and HS-UWO 241 exhibiting the highest NPQ values (Figure 2).

\section{$77 \mathrm{~K} \mathrm{Chl} \mathrm{a} \mathrm{fluorescence} \mathrm{emission} \mathrm{spectra}$}


77K emission spectra of whole cells of the mesophilic SAG 49.72 exhibited prominent fluorescence peaks at $684 \mathrm{~nm}$ and 714-716 nm consistent with LHCII-PSII and PSI fluorescence emission, respectively (Figure $3 a)$. In contrast with the mesophile, UWO 241 exhibited major Chl a fluorescence emission peaks at 685 $\mathrm{nm}$ and $697 \mathrm{~nm}$, but lacked a prominent emission peak for PSI at longer wavelengths 715-720 nm (Figure $3 \mathrm{~b})$. Acclimation to long-term stress resulted in changes in the $77 \mathrm{~K} \mathrm{Chl}$ a fluorescence emission spectra of SAG 49.72 (Figure 3a). Gaussian analysis of the fluorescence spectra revealed that SAG 49.72 exhibited a 1.5- to 3.2-fold decrease in the ratio of PSII/PSI fluorescence in response to LT, HL or HS stress (Table 3). In contrast, UWO 241 cells exhibited minimal changes in PSI fluorescence (Figure 3b), and only HS stress resulted in a minor reduction (1.18-fold relative to control) in the PSII/PSI ratio of UWO 241 (Table 3).

\section{Photosystem I energy partitioning and activity}

At the level of PSI energy partitioning, both organisms exhibited relatively high $\Phi$ PSI under all growth conditions, with the exception of HL which resulted a reduction in $\Phi$ PSI of 2.2 and 1.2-fold relative to controls in HL-SAG 49.72 and HL-UWO 241, respectively (Figure 1c,d). Both strains exhibited a concomitant increase in donor side limitation ( $\Phi N D$ ) in response to HL; however, $\Phi$ ND was higher in HL-SAG 49.72 relative to HL-UWO 241. Last, acclimation to all stress conditions resulted in lower acceptor side limitation (ФNA) relative to control conditions in UWO 241 (Figure 1d).

PSI activity was monitored in mid-log cultures of both strains acclimated to control or long-term stress conditions by far red (FR) light inducible P700 photooxidation (Figure 4). The rise in absorbance at 820 $\mathrm{nm}\left(\Delta \mathrm{A}_{820}\right)$ is a relative measure of the fraction of photooxidizable P700 reaction centers, while rates of P700 re-reduction in the dark $\left(t_{\frac{1}{2}}{ }^{\text {red }}\right)$ reflect electron donation from alternative donors and mainly CEF (A. G. Ivanov et al., 1998.). UWO 241 exhibited a significantly lower FR-inducible $\Delta \mathrm{A}_{820}$ compared to SAG 49.72. Furthermore, $\Delta \mathrm{A}_{820}$ values were below detection limits in HL-UWO 241 and HS-UWO 241 (Figure 4a). Last, UWO 241 cells grown in control and LT conditions exhibited significantly faster $t_{\frac{1}{2}}$ red compared with SAG 49.72 grown under all conditions, with the exception of $t_{\frac{1}{2}}^{\text {red }}$ from HS-SAG 49.72 (Figure 4b).

Since we were unable to detect an FR-induced $\mathrm{A}_{820}$ signal for either HL-UWO 241 or HS-UWO 241, we performed the same measurement in the presence of red actinic light (AL) after treating the cells with DCMU to block electron transfer from PSII to PSI. Under AL, we were able to measure $\mathrm{A}_{820}$ in all UWO 241 cultures (Figure 5). In agreement with the FR measurements of $\mathrm{P}_{700}$ photooxidation, acclimation to all three long-term stress conditions caused a significant reduction in $\Delta \mathrm{A}_{820}$ relative to the UWO 241 cells grown in control conditions (Figure 5a). Moreover, following AL excitation, $t_{\frac{1}{2}}^{\text {red }}$ was 1.3 to 1.6-fold faster under all stress conditions relative to that of control conditions (Figure 5b).

\section{AsA-GSH pathway}

The enzymes APX and GR are key enzymes of an ROS detoxification pathway, the AsA-GSH cycle, catalyzing the first step of the pathway and regeneration of reduced glutathione, respectively (Noctor \& Foyer, 1998). Enzymatic assays revealed low activity for either APX or GR in SAG 49.72, grown under control or HL, LT and HS stress conditions (Figure 6A). In contrast, UWO 241 exhibited significantly higher activity for both enzymes under control and all stress treatments relative to SAG 49.72 (Figure. 6). GR activity was highest in HS-UWO 241 cells, while APX activity was highest in LT-UWO 241 cells relative to controls. Last, AsA-GSH pathway substrate, ascorbate, was significantly higher in UWO 241 compared with values typically reported for other algae (Gest, Gautier, \& Stevens, 2013). Total cellular ascorbate $10.0 \pm 4.7$ and $12.3 \pm 5.71 \mathrm{mM}$ ascorbate under control and high salinity conditions, respectively (Figure S2).

Screening of the UWO 241 genome and transcriptome revealed homologs for all genes involved in the AsAGSH cycle, with the exception of monodehydroascorbate reductase (MDHAR) which was not located (Table S2). The genome of UWO 241 encodes 5 genes identified as APX (APX1, APX2-A to -D), which share a high sequence similarity with homologous genes from other photosynthetic organisms and the presence of conserved motifs involved in APX catalytic function (Figure S3a). Four of these genes (APX2-A to -D) are found on the same contig in a head-to-tail orientation and share a high sequence similarity (83.1-93.3\%), suggesting a recent gene duplication event (Figure S3b). This is in contrast with other green algae that 
typically encode one or two APX genes with confirmed APX activity (Pitsch et al., 2010; Gest et al., 2013). All other genes, including GR, were present as a single copy and shared a high sequence identity with homologous genes from C. reinhardtii (Table S2).

\section{Short-term photoinhibitory assays}

We compared the sensitivity and the recovery capacity of UWO 241 and SAG 49.72 to low temperature- and high light-induced photoinhibition by monitoring changes in the PSII photochemistry parameter, $\mathrm{F}_{\mathrm{V}} / \mathrm{F}_{\mathrm{M}}$. First, we conducted photoinhibitory assays at variable incubation temperatures $\left(2^{\circ} \mathrm{C}, 8^{\circ} \mathrm{C}, 20^{\circ} \mathrm{C}\right)$ and irradiances $\left(50 \mu \mathrm{mol} \mathrm{m} \mathrm{m}^{-2} \mathrm{~s}^{-1}, 300 \mu \mathrm{mol} \mathrm{m} \mathrm{m}^{-2} \mathrm{~s}^{-1}, 1000 \mu \mathrm{mol} \mathrm{m} \mathrm{m}^{-2} \mathrm{~s}^{-1}\right)$. Decline in $\mathrm{F}_{\mathrm{V}} / \mathrm{F}_{\mathrm{M}}$ was recorded at various time points over $3 \mathrm{~h}$ (Figure S4). At $8^{\circ} \mathrm{C}$ and $20^{\circ} \mathrm{C}$ incubation temperatures, UWO 241 exhibited higher sensitivity to both medium and high light treatments, relative to SAG 49.72. However, when the assay temperature was decreased to $2^{\circ} \mathrm{C}$, SAG 49.72 exhibited higher photoinhibition under low light, while both strains exhibited comparable sensitivity to medium or high light treatment (Figure S4). Thus, for the recovery assays, we choose to test samples for $1 \mathrm{hr}$ under two photoinhibitory treatments: i) low temperature (LT, $2^{\circ} \mathrm{C}$ ) and ii) high light (HL, $\left.300 \mu \mathrm{mol} \mathrm{m} \mathrm{m}^{-2} \mathrm{~s}^{-1}\right)$. In agreement with our results from the preliminary assays, UWO 241 and SAG 49.72 exhibited differential sensitivity to HL and LT treatments, respectively. $1 \mathrm{hr}$ of HL-treatment resulted in $62 \%$ loss of $\mathrm{F}_{\mathrm{V}} / \mathrm{F}_{\mathrm{M}}$ in UWO 241 , while LT caused a $32 \%$ reduction in $\mathrm{F}_{\mathrm{V}} / \mathrm{F}_{\mathrm{M}}$ in SAG 49.72 (Figure 7a). LT and HL treatment cause $<10 \%$ reduction in $\mathrm{F}_{\mathrm{V}} / \mathrm{F}_{\mathrm{M}}$ in UWO 241 and SAG 49.72, respectively. In addition, HL-treatment of UWO 241 resulted in almost complete reduction in qL which was accompanied by a 7 -fold increase in NPQ. LT-treated SAG 49.72 exhibited only a $67 \%$ reduction in qL. Both strains exhibited comparable increases in NPQ in response to the LT treatment (Figure 7b).

Despite the strain differences in sensitivity to short-term photooxidative stress, both organisms exhibited full recovery to initial $\mathrm{F}_{\mathrm{V}} / \mathrm{F}_{\mathrm{M}}$ levels within $2 \mathrm{~h}$ (Figure 7a). The recovery was most dramatic in HL-treated UWO 241 which exhibited a rapid rate of recovery within the first 15 mins (Figure 7A). Strain- and treatmentspecific differences were observed at the level of recovery of qL and NPQ. HL-treated cells of UWO 241 were unable to restore to initial qL levels, and $\mathrm{qL}$ remained close to zero even 2 hours of recovery (Figure 7b). Similarly, NPQ also remained high in HL-treated UWO 241 during the recovery phase (Figure 7c). In contrast, both strains exhibited similar trends in relaxation of NPQ following LT treatment (Figure 7c).

Last, we monitored whether short-term HL- or LT-treatments were accompanied by accumulation of an ROS, using the dye NBT as a semi-quantitative measurement of $\mathrm{O}_{2}{ }^{-}$(Figure 8). The mesophile SAG 49.72 produced higher levels of $\mathrm{O}_{2}{ }^{-}$relative to pre-treated cells under both HL and LT treatments. In marked contrast, UWO 241 maintained very low levels of $\mathrm{O}_{2}{ }^{-}$under either short-term HL or LT stress (Figure 8).

\section{DISCUSSION}

This study examined whether two Chlamydomonas species adapted to extreme contrasts in their native environments rely upon comparable strategies for survival under long-term stress conditions. SAG 49.72 was originally isolated from a temperate lake: it is a mesophilic species and possesses limited ability to acclimate to either salinity or low temperature stress (Pocock et al., 2011; Szyszka et al., 2007). In contrast, in its native Antarctic lake environment, UWO 241 has survived under permanent low temperature and hypersalinity stress for at least 1000 years, based on estimates of the last occurrence of ice-free conditions in Lake Bonney (Morgan-Kiss et al., 2006). Our results confirmed that although both the mesophilic SAG 49.72 and the psychrophilic UWO 241 exhibited the ability to grow robustly under high light, low temperature or high salinity, their tolerance levels and long-term acclimatory strategies to these environmental stressors were markedly different. For the mesophilic SAG 49.72, long-term acclimation could be summarized into maintenance of photostasis by adjustments in PSII antenna size and PSII-PSI energy distribution, both classic long-term acclimatory mechanisms described for other model algal species (Maxwell, Falk, Trick, \& Hüner, 1994; Tanaka \& Melis, 1997). In contrast, the psychrophilic UWO 241 relies upon constitutive CEF and continuous ROS detoxification capacity to provide photoprotection of PSII and PSI under long-term stress.

Long-term stress acclimation in the mesophile SAG 49.72 involved an increase in the ratio of $\mathrm{Chl} \mathrm{a} / \mathrm{b}$ and a 
concomitant decrease in PSII/PSI at the level of 77K fluorescence emission. Higher Chl a/b ratios in response to long-term stress have been reported across many algae and plants and coincides with a reduction in the size of LHCII (Maxwell et al., 1994; Smith et al., 1990; Wilson \& Hüner, 2000). Reductions in PSII/PSI stoichiometry under either high light or low temperature stress are also well documented and reflects enhanced distribution of absorbed light energy in favor of PSI (Smith et al., 1990; Velitchkova, Popova, Faik, Gerganova, \& Ivanov, 2020). In contrast with SAG 49.72, UWO 241 does not appear to rely on either of these classic acclimatory mechanisms to survive long-term stress. Morgan-Kiss et al. (2002) demonstrated that UWO 241 is also unable to undergo state transitions. More recently, Szyska-Mroz and colleagues reported that the psychrophile relies instead on a poorly understood constitutive spill-over mechanism under HS growth conditions (Szyszka-Mroz et al., 2019). Thus, UWO 241 is a natural variant lacking state transitions that maintains a relatively large LHCII and high PSII content under long-term stress. Despite the lack of these well-characterized mechanisms, UWO 241 maintained a high qL and comparable energy partitioning relative to control conditions, suggesting that the psychrophile relies on alternative processes to avoid high excitation pressure.

CEF is an essential process in plants and algae for balancing ATP/NADPH and photoprotection; although, most studies have considered the role of CEF under short-term stress. Early reports identified that UWO 241 exhibits relatively high rates of PSI-driven CEF compared with mesophilic strains (Morgan-Kiss et al., 2002b; Morgan-Kiss et al., 2006). Maximal CEF requires restructuring of the photosynthetic apparatus and assembly of a novel PSI supercomplex (Kalra et al., 2020; Szyszka-Mroz et al., 2015). The UWO 241 supercomplex is distinct from that of previously described complex from C. reinhardtii because the former is not associated with state-transition-inducing treatments and it lacks typical PSI 77K fluorescence emission despite the presence of many PSI core proteins (Iwai et al., 2010; Kalra et al., 2020; Szyszka-Mroz et al., 2015). Here we show that UWO 241 exhibits faster CEF rates under salinity stress, high light or low temperatures, suggesting that the extremophile relies on CEF as a general long-term acclimatory strategy.

CEF generates additional transthylakoid proton motive force which has been proposed to be utilized for several purposes, including balancing ATP/NADPH production and photoprotection of both PSII and PSI (Bulte, Gans, Febeille, \& Wollman, 1990; Chaux, Peltier, \& Johnson, 2015; He et al., 2015; Lucker \& Kramer, 2013; Yamori et al., 2016). Kalra and colleagues showed that under long-term HS stress CEF serves multiple purposes in UWO 241, including additional ATP production as well as constitutive photoprotection (Kalra et al., 2020). Higher ATP levels are used in part to support enhanced CBB pathway activity which supplies substrates for storage compounds (starch), osmoregulants (glycerol), as well as the shikimate pathway (Kalra et al., 2020). It is likely that CEF is utilized for similar processes when UWO 241 is acclimated to HL or LT. This current study provides evidence that high CEF in all three stress conditions is associated with enhanced photoprotection of PSII. Increased CEF rates in cells of UWO 241 acclimated to HL, LT or HS all exhibited a higher capacity for NPQ compared with control cells. This suggests constitutive capacity for PSII protection owing to enhanced CEF-generated pmf.

High CEF provides PSI photoprotection. UWO 241 cultures acclimated to all three long-term stress conditions were associated with reduced levels of FNA relative to control-grown UWO 241 cells. These data suggest that CEF also contributes to protection of PSI by preventing accumulation of reduced Fd and minimizing acceptor side limitation. Over-reduction of PSI manifests as production of the ROS, $\mathrm{O}_{2}^{-}$(Asada, 1999). We show that UWO 241 appears to possess remarkable ability to avoid $\mathrm{O}_{2}{ }^{-}$accumulation: cells exposed to either short-term LT or HL stress exhibited minimal accumulation of this ROS. This ability to keep $\mathrm{O}_{2}^{-}$levels lows is likely due to CEF-associated prevention of PSI acceptor side limitation. In contrast the mesophile exhibited significant levels of $\mathrm{O}_{2}{ }^{-}$when exposed to the same conditions. While PSII is typically considered sensitive to all environmental stresses, PSI photodamage occurs under specific environmental conditions, including drought, high salinity and low temperature, and repair of PSI is slow and inefficient (Huang, Yang, Zhang, Zhang, \& Cao, 2012; Huang, Yang, Hu, \& Zhang, 2016; Huang, Zhang, Xu, \& Liu, 2017; A. G. Ivanov et al., 1998; Yamori et al., 2016; Zhang \& Scheller, 2004). Thus, PSI photoinhibition is a deleterious consequence for survival under long-term stress. We suggest that constitutive CEF simultaneously plays critical roles in protecting both PSII and PSI from photo-damage in UWO 241 for survival under long-term 
environmental stress.

UUWO 241 exhibits constitutive protection of PSII and PSI in UWO 241 by minimizing ROS production; however, there is also evidence that the psychrophile possesses enhanced ability for ROS detoxification. The AsA-GSH pathway is a major ROS detoxification pathway in plants, and is responsible for regeneration of the antioxidant ascorbate (Foyer \& Noctor, 2012; Foyer \& Shigeoka, 2011). The AsA-GSH pathway involves four enzymes, ascorbate peroxidase (APX), monohydroascorbate reductase (MDHAR), dehydroascorbate reductase (DHAR), and glutathione reductase (GR) (Noctor \& Foyer, 1998). Plants express multiple isoforms of each enzyme, in particular APX (Pitsch et al. 2010; Teixeira, Menezes-Benavente, Margis, \& MargisPinheiro, 2004). High concentrations of ascorbate accumulate in plants, particularly under stress conditions, including high light, low temperatures and high salinity (Bartoli, Buet, Grozeff, Galatro, \& Simontacchi, 2017; Maruta \& Ishikawa, 2017; Wildi \& Lütz, 1996; Zechmann, Stumpe, \& Mauch, 2011; Zhang et al., 2011). On the other hand, cyanobacteria and algae exhibit significantly lower levels of ascorbate and possess only one isoform or are missing one or more enzymes of the AsA-GSH pathway (Gest et al., 2013). For example, the model $C$. reinhardtii appears to lack the thylakoid-bound APX found in plants, expressing only a single isoform of APX which is localized to the stroma (Pitsch et al., 2010). A second APX2 isoform has been predicted to localize to the chloroplast, but its function has not been studied (Wu and Wang, 2019). Three pieces of evidence indicate that UWO 241 may rely on the AsA-GSH pathway to a greater extent than previously appreciated in other algal species. First, activity of two enzymes, APX and GR, are constitutively high in UWO 241 relative to the mesophile SAG 49.72 under both control and all long-term stress conditions. Second, UWO 241 cells accumulated millimolar levels of the substrate ascorbate. Last, unlike other algae studied thus far, UWO 241 appears to possess more isoforms of several enzymes necessary for ascorbate cycling. A search of a previously published transcriptome of UWO 241 (Raymond \& Morgan-Kiss, 2013) revealed multiple potential homologues for enzymes of the AsA pathway, including 3 APX, 3 DHAR, and 3 GR genes (Table S1). These genes were also detected at the level of the genome, with four APX genes present as highly similar tandem duplicates (Table S2; Figure S3). In addition, one of the putative UWO 241 APX proteins is related to a plant thylakoid-bound isoform from Triticum aestivum . APX catalyzes the oxidation of ascorbate by $\mathrm{H}_{2} \mathrm{O}_{2}$, while DHAR and GR work in concert to regenerate glutathione. We did not identify an isoform for MDHAR in the genome or transcriptome, which is needed for recycling of ascorbate. The additional isoforms may be localized to different cellular compartments, as in plants, or may contribute to constitutively high AsA-GSH pathway activity. Gene duplications have been shown for several other UWO 241 genes including photosynthetic ferredoxin (Cvetkovska et al., 2018), chlorophyllide a oxygenase (Cvetkovska et al., 2019) and the chloroplast kinase Stl-1 (Szyszka-Mroz et al., 2019).

\section{Conclusions and renaming of UWO 241}

This study builds upon more than two decades of work on the enigmatic, Antarctic alga, Chlamydomonas sp. UWO 241 which have documented novel adaptation strategies to survive permanent extreme conditions. The taxonomic identity of UWO 241 has been the subject of debate: originally identified on a morphological basis as C. subcaudata by Priscu \& Neale (1995), the organism was erroneously identified as $C$. raudensis UWO 241 (Pocock et al., 2004). Recently, a thorough revisiting on the taxonomy of the strain performed by Possmayer et al. (Possmayer et al., 2016) concluded that UWO 241 represents a unique lineage within the Moewusinia clade, and it was therefore renamedChlamydomonas sp. UWO 241 as a place holder. Molecular phylogenetic analysis of the full length $18 \mathrm{~S}$ rRNA gene revealed that the closest known relative of UWO 241 is a marine alga, Chlamydomonas parkeae SAG 24.89 (95\% identity). Given the geographical isolation and unique physiology, combined with the molecular and genomic analyses, we suggest that UWO 241 is a unique strain. According to requirements of the International Code of Nomenclature for algae, fungi, and plants (McNeill et al., 2012) we propose to rename the strain Chlamydomonas priscuii in recognition of John C. Priscu, the investigator who originally isolated the strain in 1995 (Neale \& Priscu, 1995).

\section{ACKNOWLEDGEMENTS}

The authors thank Prof. John C. Priscu (Montana State University) for isolation and donation of the algal strain Chlamydomonas sp. UWO 241 (originally named Chlamydomonas subcaudata ) from Lake Bonney, 
McMurdo Dry Valleys, Antarctica. The authors thank A. Mills and J. Prutz for assistance with growing cultures and ROS measurements, respectively. RMK, SS, JP and MH were supported by the National Science Foundation, Office of Polar Programs under award \#OPP-1056396 (growth physiology, photoinhibition, antioxidant measurements). IK, AM and SD were supported by the U.S. Department of Energy (DOE), Office of Science, Basic Energy Sciences (BES) under award \#DE-SC0019138 (PSI, CEF measurements). MC is supported by a National Sciences and Engineering Research Council of Canada (NSERC) Discovery Grant RGPIN-2019-05763 and University of Ottawa start-up funding (genome screening).

\section{REFERENCES}

Aldesuquy, H. S., Baka, Z. A., El-Shehaby, O., \& Ghanem, H. E. (2013). Growth, Lipid peroxidation and antioxidant enzyme activities as a Selection Criterion for the salt tolerance of wheat cultivars irrigated by seawater. Phyton, 53 , 153-165.

Apel, K., \& Hirt, H. (2004). Reactive oxygen species: Metabolism, oxidative stress, and signal transduction. Annual Review of Plant Biology, 55 , 373-399.

Asada, K. (1996). Photosynthesis and the Environment. by NR Baker, Kluwer, 123.

Asada, K. (1999). The waterwater cycle in chloroplasts: scavening of active oxygens and dissipation of excess photon. Annual Review of Plant Physiology 83 Plant Molecular Biology, 50 , 601-639.

Asada, K. (2000). The water-water cycle as alternative photon and electron sinks. Philosophical Transactions of the Royal Society of London. Series B: Biological Sciences, 355 (1402), 1419-1431.

Asada, K. (2006). Production and scavenging of reactive oxygen species in chloroplasts and their functions. Plant Physiology, 141 (2), 391-396.

Bartels, D., \& Sunkar, R. (2005). Drought and salt tolerance in plants. Critical Reviews in Plant Sciences, $24(1), 23-58$.

Bartoli, C. G., Buet, A., Grozeff, G. G., Galatro, A., \& Simontacchi, M. (2017). Ascorbate-glutathione cycle and abiotic stress tolerance in plants. In Ascorbic acid in plant growth, development and stress tolerance (pp. 177-200): Springer.

Bulte, L., Gans, P., Febeille, F., \& Wollman, F. (1990). ATP control on state transitions in vivo in Chlamydomonas reinhardtii . Biochimica et Biophysica Acta, 1020 , 72-80.

Chaux, F., Peltier, G., \& Johnson, X. (2015). A security network in PSI photoprotection: regulation of photosynthetic control, NPQ and O2 photoreduction by cyclic electron flow. Frontiers in Plant Science, 6 .

Chen, Q., Zhang, M., \& Shen, S. (2011). Effect of salt on malondialdehyde and antioxidant enzymes in seedling roots of Jerusalem artichoke (Helianthus tuberosus L.).Acta Physiologiae Plantarum, 33 (2), 273278.

Cook, G., Teufel, A., Kalra, I., Li, W., Wang, X., Priscu, J., \& Morgan-Kiss, R. J. P. r. (2019). The Antarctic psychrophiles Chlamydomonas spp. UWO241 and ICE-MDV exhibit differential restructuring of photosystem I in response to iron.Photosynthesis Research , 141, 209-228.

Cournac, L., Latouche, G., Cerovic, Z., Redding, K., Ravenel, J., \& Peltier, G. (2002). In vivo interactions between photosynthesis, mitorespiration, and chlororespiration in Chlamydomonas reinhardtii . Plant Physiology, 129 (4), 1921-1928.

Cvetkovska, M., Hüner, N. P., \& Smith, D. R. (2017). Chilling out: the evolution and diversification of psychrophilic algae with a focus on Chlamydomonadales. Polar Biology, 40 , 1169-1184.

Ensminger, I., Busch, F., \& Hüner, N. P. A. (2006). Photostasis and cold acclimation: sensing low temperature through photosynthesis. Physiologia Plantarum, 126 (1), 28-44. 
Falk, S., Krol, M., Maxwell, D. P., Rezansoff, D. A., Gray, G. R., \& Hüner, N. P. A. (1994). Changes in in vivo fluorescence quenching in rye and barley as a function of reduced PSII light harvesting antenna size. Physiologia Plantarum, 91 , 551-558.

Falk, S., Maxwell, D., Gray, G., Rezansoff, D., \& Hüner, N. (1993). Photosynthetic acclimation to low temperature in higher plants and algae. Current Topics in Botanical Research, 1 , 281-292.

Förster, B., Osmond, C. B., \& Pogson, B. J. (2005). Improved survival of very high light and oxidative stress is conferred by spontaneous gain-of-function mutations inChlamydomonas . Biochimica et Biophysica Acta (BBA) - Bioenergetics, 1709 (1), 45-57.

Foyer, C. H., \& Halliwell, B. (1976). The presence of glutathione and glutathione reductase in chloroplasts: a proposed role in ascorbic acid metabolism. Planta, 133 (1), 21-25.

Foyer, C. H., Lopez-Delgado, H., Dat, J. F., \& Scott, I. M. (1997). Hydrogen peroxide-and glutathioneassociated mechanisms of acclimatory stress tolerance and signalling. Physiologia Plantarum, 100 (2), 241254.

Foyer, C. H., \& Noctor, G. (2012). Managing the cellular redox hub in photosynthetic organisms.Plant, Cell \& Environment, 35 (2), 199-201.

Foyer, C. H., \& Shigeoka, S. (2011). Understanding oxidative stress and antioxidant functions to enhance photosynthesis. Plant Physiology, 155 (1), 93-100.

Gest, N., Gautier, H., \& Stevens, R. (2013). Ascorbate as seen through plant evolution: the rise of a successful molecule? Journal of Experimental Botany, 64 (1), 33-53.

He, Y., Fu, J., Yu, C., Wang, X., Jiang, Q., Hong, J., . . . James, A. (2015). Increasing cyclic electron flow is related to $\mathrm{Na}+$ sequestration into vacuoles for salt tolerance in soybean. Journal of Experimental Botany , erv392.

Hu, W., Song, X., Shi, K., Xia, X., Zhou, Y., \& Yu, J. (2008). Changes in electron transport, superoxide dismutase and ascorbate peroxidase isoenzymes in chloroplasts and mitochondria of cucumber leaves as influenced by chilling. Photosynthetica, 46 (4), 581 .

Huang, W., Yang, S.-J., Zhang, S.-B., Zhang, J.-L., \& Cao, K.-F. (2012). Cyclic electron flow plays an important role in photoprotection for the resurrection plantParaboea rufescens under drought stress. Planta, 235 (4), 819-828.

Huang, W., Yang, Y.-J., Hu, H., \& Zhang, S.-B. (2016). Seasonal variations in photosystem I compared with photosystem II of three alpine evergreen broad-leaf tree species. Journal of Photochemistry and Photobiology B: Biology, 165 , 71-79.

Huang, W., Zhang, S.-B., Xu, J.-C., \& Liu, T. (2017). Plasticity in roles of cyclic electron flow around photosystem I at contrasting temperatures in the chilling-sensitive plant Calotropis gigantea . Environmental and Experimental Botany, 141, 145-153.

Huner, N., Dahal, K., Hollis, L., Bode, R., Rosso, D., Krol, M., \& Ivanov, A. G. (2012). Chloroplast redox imbalance governs phenotypic plasticity: the "grand design of photosynthesis" revisited. Frontiers in Plant Science, $3,255$.

Ivanov, A., Sane, P., Simidjiev, I., Park, Y.-I., Huner, N., \& Oquist, G. (2012). Restricted capacity for PSI-dependent cyclic electron flow in $\Delta$ petE mutant compromises the ability for acclimation to iron stress in Synechococcus sp. PCC 7942 cells. Biochimica et Biophysica Acta, 1817 (8), 1277-1284.

Ivanov, A. G., Morgan, R. M., Gray, G. R., Velitchkova, M. Y., \& Hüner, N. P. (1998). Temperature/light dependent development of selective resistance to photoinhibition of photosystem I. FEBS Letters 430 (3), 288-292. 
Iwai, M., Takizawa, K., Tokutsu, R., Okamuro, A., Takahashi, Y., \& Minagawa, J. (2010). Isolation of the elusive supercomplex that drives cyclic electron flow in photosynthesis.Nature, 464 (7292), 1210-1213.

Jeffrey, S. W., \& Humphrey, G. F. (1975). New spectrophotometric equations for determining chlorophyll a, b, c1, c2 in higher plants, algae and natural phytoplankton.Biochem. Physiol. Pflanz, 167, 191-194.

Julkowska, M. (2020). Extreme Engineering: How Antarctic Algae Adapt to Hypersalinity. Plant Physiology, 183 (2), 427.

Kalra, I., Wang, X., Cvetkovska, M., Jeong, J., McHargue, W., Zhang, R., . . Morgan-Kiss, R. M. (2020). Chlamydomonas sp. UWO 241 exhibits high cyclic electron flow and rewired metabolism under high salinity. Plant Physiology, 183 , 588-601.

Ledford, H. K., Chin, B. L., \& Niyogi, K. K. (2007). Acclimation to singlet oxygen stress in Chlamydomonas reinhardtii. Eukaryot Cell, 6 . doi:10.1128/ec.00207-06

Liu, Y., Qi, M., \& Li, T. (2012). Photosynthesis, photoinhibition, and antioxidant system in tomato leaves stressed by low night temperature and their subsequent recovery.Plant Science, 196 , 8-17.

Lucker, B., \& Kramer, D. M. (2013). Regulation of cyclic electron flow in Chlamydomonas reinhardtiiunder fluctuating carbon availability. Photosynthesis Research, 117 (1-3), 449-459.

Maruta, T., \& Ishikawa, T. (2017). Ascorbate peroxidases: crucial roles of antioxidant enzymes in plant stress responses. In Ascorbic acid in plant growth, development and stress tolerance (pp. 111-127): Springer.

Maxwell, D. P., Falk, S., Trick, C. G., \& Hüner, N. P. A. (1994). Growth a low temperature mimics high-light acclimation in Chlorella vulgaris . Plant Physiology, 105, 535-543.

McNeill, J., Barrie, F., Buck, W., Demoulin, V., Greuter, W., Hawksworth, D., . . . Prado, J. (2012). International Code of Nomenclature for algae, fungi and plants. Regnum vegetabile, 154 .

Minagawa, J. (2011). State transitions-the molecular remodeling of photosynthetic supercomplexes that controls energy flow in the chloroplast. Biochimica et Biophysica Acta (BBA)-Bioenergetics, 1807 (8), 897905.

Møller, I. M., Jensen, P. E., \& Hansson, A. (2007). Oxidative modifications to cellular components in plants. Annual Reviews of Plant Biology, 58 , 459-481.

Morgan-Kiss, R., Ivanov, A. G., Williams, J., Mobashsher, K., \& Hüner, N. P. (2002a). Differential thermal effects on the energy distribution between photosystem II and photosystem I in thylakoid membranes of a psychrophilic and a mesophilic alga. Biochimica et Biophysica Acta (BBA) - Bioenergetics, 1561 (2), 251-265.

Morgan-Kiss, R. M., Ivanov, A. G., \& Hüner, N. P. A. (2002b). The Antarctic psychrophile, Chlamydomonas subcaudata, is deficient in state I-state II transitions. Planta, 214 (3), 435-445.

Morgan-Kiss, R. M., Ivanov, A. G., Modla, S., Czymmek, K., Hüner, N. P., Priscu, J. C., . . Hanson, T. E. (2008). Identity and physiology of a new psychrophilic eukaryotic green alga, Chlorella sp., strain BI, isolated from a transitory pond near Bratina Island, Antarctica. Extremophiles, 12 (5), 701-711.

Morgan-Kiss, R. M., Priscu, J. C., Pocock, T., Gudynaite-Savitch, L., \& Hüner, N. P. (2006). Adaptation and acclimation of photosynthetic microorganisms to permanently cold environments. Microbiology $\&$ Molecular Biology Reviews, 70 (1), 222-252.

Morgan, R. M., Ivanov, A. G., Priscu, J. C., Maxwell, D. P., \& Hüner, N. P. A. (1998). Structure and composition of the photochemical apparatus of the Antarctic green alga, Chlamydomonas subcaudata. Photosynthesis Research, 56 , 303-314.

Müller, P., Li, X.-P., \& Niyogi, K. K. (2001). Non-photochemical quenching. A response to excess light energy. Plant Physiology, 125 (4), 1558-1566. 
Neale, P. J., \& Priscu, J. C. (1995). The photosynthetic apparatus of phytoplankton from a perennially ice-covered Antarctic lake: acclimation to an extreme shade environment.Plant and Cell Physiology, 36 , 253-263.

Nichols, H. W., \& Bold, H. C. (1965). Trichosarcina polymorpha Gen. Et Sp. Nov. J Phycol., 1 , 34-38.

Niyogi, K. K. (1999). Photoprotection revisited: genetic and molecular approaches. Annual Review of Plant Biology, 50 (1), 333-359.

Noctor, G., \& Foyer, C. H. (1998). Ascorbate and glutathione: keeping active oxygen under control.Annual Review of Plant Biology, 49 (1), 249-279.

Öquist, G., \& Hüner, N. P. (2003). Photosynthesis of overwintering evergreen plants. Annual Review Plant Biology, 54, 329-355.

Pitsch, N. T., Witsch, B., \& Baier, M. (2010). Comparison of the chloroplast peroxidase system in the chlorophyte Chlamydomonas reinhardtii, the bryophytePhyscomitrella patens, the lycophyte Selaginella moellendorffi $i$ and the seed plant Arabidopsis thaliana .BMC Plant Biology, 10 (1), 133.

Pocock, T., Lachance, M.-A., Proschold, T., Priscu, J. C., Kim, S., \& Huner, N. P. A. (2004). Identification of a psychrophilic green alga from Lake Bonney Antarctica: Chlamydomonas raudensis ETTL. (UWO 241) (Chlorophyceae ). Journal of Phycology, 40 , 1138-1148.

Pocock, T., Koziak, A., Rosso, D., Falk, S., \& Hüner, H. P. A. (2007). Chlamydomonas raudensisettl. (UWO241) exhibits the capacity for rapid D1 repair in response to chronic photoinhibition at low temperature. Journal of Phycology, 43, 924-936.

Pocock, T., Vetterli, A., \& Falk, S. (2011). Evidence for phenotypic plasticity in the Antarctic extremophileChlamydomonas raudensis Ettl. UWO 241. J Experimental Botany, 62 (3), 1169-1177. doi:10.1093/jxb/erq347

Possmayer, M., Gupta, R. K., Szyszka-Mroz, B., Maxwell, D. P., Lachance, M. A., Huner, N., \& Smith, D. R. (2016). Resolving the phylogenetic relationship between Chlamydomonas sp. UWO 241 and Chlamydomonas raudensis sag 49.72 (Chlorophyceae) with nuclear and plastid DNA sequences. Journal of Phycology, 52 (2), 305-310.

Raymond, J. A., \& Morgan-Kiss, R. (2013). Separate Origins of ice-binding proteins in Antarctic Chlamydomonas species. PLoS ONE, 8 (3), e59186.

Schreiber, U., \& Klughammer, C. (2008). Non-photochemical fluorescence quenching and quantum yields in PS I and PS II: analysis of heat-induced limitations using Maxi-Imaging-PAM and Dual-PAM-100. PAM Application Notes, 1, 15-18.

Sharma, P., Jha, A. B., Dubey, R. S., \& Pessarakli, M. (2012). Reactive oxygen species, oxidative damage, and antioxidative defense mechanism in plants under stressful conditions. Journal of Botany, 2012 .

Sirikhachornkit, A., \& Niyogi, K. K. (2010). Antioxidants and Photo-oxidative Stress Responses in Plants and Algae. In Govindjee \& T. D. Sharkey (Eds.), Advances in Photosynthesis and Respiration (Vol. 31, pp. 379-396): Springer Netherlands.

Smith, B. M., Morrissey, P. J., Guenther, J. E., Nemson, J. A., Harrison, M. A., Allen, J. F., \& Melis, A. (1990). Response of the photosynthetic apparatus in Dunaliella salina (green algae) to irradiance stress. Plant Physiology, 93 (4), 1433-1440.

Suzuki, N., Koussevitzky, S., Mittler, R., \& Miller, G. (2012). ROS and redox signalling in the response of plants to abiotic stress. Plant, Cell $\&$ Environment, 35 (2), 259-270.

Szyszka-Mroz, B., Cvetkovska, M., Ivanov, A. G., Smith, D. R., Possmayer, M., Maxwell, D. P., \& Huner, N. P. (2019). Cold-adapted protein kinases and thylakoid remodeling impact energy distribution in an Antarctic 
psychrophile. Plant Physiology, 180 (3), 1291-1309.

Szyszka-Mroz, B., Pittock, P., Ivanov, A. G., Lajoie, G., \& Huner, N. P. (2015). The Antarctic psychrophile, Chlamydomonas sp. UWO 241, preferentially phosphorylates a PSI-cytochrome b6/f supercomplex. Plant Physiology, 169 , 717-736.

Szyszka, B., Ivanov, A. G., \& Huner, N. P. (2007). Psychrophily is associated with differential energy partitioning, photosystem stoichiometry and polypeptide phosphorylation in Chlamydomonas raudensis. Biochimica et Biophysica Acta (BBA)-Bioenergetics, 1767 (6), 789-800.

Szyszka, B., Ivanov, A. G., \& Huner, N. P. A. (2007). Psychrophily induces differential energy partitioning, photosystem stoichiometry and polypeptide phosphorylation inChlamydomonas raudensis . Biochimica et Biophysica Acta (BBA) - Bioenergetics, 1767 , 789-800.

Takahashi, S., \& Murata, N. (2008). How do environmental stresses accelerate photoinhibition? Trends in Plant Science, 13 (4), 178-182.

Tanaka, A., \& Melis, A. (1997). Irradiance-dependent changes in the size and composition of the chlorophyll $a-b$ light-harvesting complex in the green algaDunaliella salina . Plant Cell Physiolgy, 38 (1), 17-24.

Teixeira, F. K., Menezes-Benavente, L., Margis, R., \& Margis-Pinheiro, M. (2004). Analysis of the molecular evolutionary history of the ascorbate peroxidase gene family: inferences from the rice genome. J. Mol. Evol., 59 (6), 761-770.

Van Alstyne, K. L., Sutton, L., \& Gifford, S.-A. (2020). Inducible versus constitutive antioxidant defenses in algae along an environmental stress gradient. Marine Ecology Progress Series, 640 , 107-115.

Velitchkova, M., Popova, A. V., Faik, A., Gerganova, M., \& Ivanov, A. G. (2020). Low temperature and high light dependent dynamic photoprotective strategies in Arabidopsis thaliana. Physiologia Plantarum .

Venisse, J.-S., Gullner, G., \& Brisset, M.-N. (2001). Evidence for the involvement of an oxidative stress in the initiation of infection of pear by Erwinia amylovora.Plant Physiology, 125 (4), 2164-2172.

Wildi, B., \& Lutz, C. (1996). Antioxidant composition of selected high alpine plant species from different altitudes. Plant, Cell \& Environment, 19 (2), 138-146.

Wilson, K. E., \& Huner, N. P. (2000). The role of growth rate, redox-state of the plastoquinone pool and the trans-thylakoid $\Delta \mathrm{pH}$ in photoacclimation of Chlorella vulgaris to growth irradiance and temperature. Planta, 212 (1), 93-102.

Witman, G. B. (1993). Chlamydomonas phototaxis. Trends in cell biology, 3 (11), 403-408.

Yamori, W., Makino, A., \& Shikanai, T. (2016). A physiological role of cyclic electron transport around photosystem I in sustaining photosynthesis under fluctuating light in rice. Scientific reports, 6 , 20147.

Young, J. N., \& Schmidt, K. (2020). It's what's inside that matters: physiological adaptations of highlatitude marine microalgae to environmental change. New Phytologist .

Zechmann, B., Stumpe, M., \& Mauch, F. (2011). Immunocytochemical determination of the subcellular distribution of ascorbate in plants. Planta, 233 (1), 1-12.

Zhang, C., Liu, J., Zhang, Y., Cai, X., Gong, P., Zhang, J., . . . Ye, Z. (2011). Overexpression of SlGMEs leads to ascorbate accumulation with enhanced oxidative stress, cold, and salt tolerance in tomato. Plant cell reports, 30 (3), 389-398.

Zhang, S., \& Scheller, H. V. (2004). Photoinhibition of photosystem I at chilling temperature and subsequent recovery in Arabidopsis thaliana. Plant and Cell Physiology, 45 (11), 1595-1602.

\section{FIGURE LEGENDS}


FIGURE 1. Effect of long-term stress acclimation on partitioning of PSII (a) and PSI (b) in UWO 241 and SAG 49.72 grown under control and long term stress conditions. $\Phi$ NO, non-regulated energy dissipation; $\Phi$ NPQ, non-photochemical energy quenching; PPSII, PSII quantum yield; $\Phi P S I$, PSI quantum yield; $\Phi$ ND, donor side limitation; $\Phi$ NA, acceptor side limitation. C, Control; HL, High Light; LT, Low Temperature; HS, High Salt. Values are means with standard deviations $(n=3)$.

FIGURE 2. Capacity for nonphotochemical quenching in UWO 241 (UWO) and SAG 49.72 (SAG) grown under control and high stress conditions. C, Control; HL, high light; LT, low temperature; HS, high salt. Letters - a, statistical significance between control vs. stress within one algal species; b, statistical significance between UWO 241 vs. SAG 49.72 when grown under same treatment $(\mathrm{n}=3 ; \mathrm{p}<0.05)$.

FIGURE 3. $77 \mathrm{~K} \mathrm{Chl} \mathrm{a} \mathrm{fluorescence} \mathrm{emission} \mathrm{spectra} \mathrm{of} \mathrm{the} \mathrm{mesophile} \mathrm{SAG} 49.72$ (a) and the psychrophile UWO 241 (b) and acclimated to control and long-term stress treatments. C, Control; HL, high light; LT, low temperature; HS, high salt.

FIGURE 4. Photosystem I (P700) oxidation/reduction of the mesophile SAG 49.72 and the psychrophile UWO 241 grown under control and long-term stress conditions. a. Oxidation state of $\mathrm{P}_{700}$. b. Re-reduction kinetics of $\mathrm{P}_{700}+$. $\mathrm{P}_{700}$ oxidation/reduction was monitored in the presence of far red light. Letters - a, statistical significance between control vs. stress within one algal species; b, statistical significance between UWO 241 vs. SAG 49.72 when grown under same treatment $(\mathrm{n}=3 ; \mathrm{p}<0.05)$. C, Control; HL, high light; LT, low temperature; HS, high salt; ND, not detectable.

FIGURE 5. Photosystem I oxidation/reduction kinetics in UWO 241 grown under control and long-term stress conditions. $\mathrm{P}_{700}$ oxidation/reduction was monitored in the presence of red actinic light following DCMU pretreatment. (a) Oxidation state of $\mathrm{P}_{700}$. (b) Re-reduction kinetics of $\mathrm{P}_{700}+$. Letters statistical significance between control vs. stress $(\mathrm{n}=3 ; \mathrm{p}<0.05)$. C, Control; HL, high light; LT, low temperature; HS, high salt.

FIGURE 6. Activity of AsA-GSH pathway enzymes, ascorbate peroxidase (APX, a) and glutathione reductase (GR, b) the mesophile SAG 49.72 and the psychrophile UWO 241 grown under control and longterm stress conditions. Letters - a, statistical significance between control vs. stress within one algal species; b, statistical significance between UWO 241 vs. SAG 49.72 when grown under same treatment $(\mathrm{n}=3 ; \mathrm{p}<0.05)$. C, Control; HL= high light; LT, low temperature; HS, high salt.

FIGURE 7. Photoinhibition and recovery in the mesophile SAG 49.72 and the psychrophile UWO 241. Cultures grown under control conditions exposed to either high light (HL, $300 \mu \mathrm{mol} \mathrm{m}{ }^{-2} \mathrm{~s}^{-1}$ ) or low temperature LT, $\left(2^{\circ} \mathrm{C}\right)$ short-term stress for $60 \mathrm{~min}$ then returned to control conditions for $120 \mathrm{~min}$ to recover. (a) Percent photoinhibition was determined by loss of photochemical ability $\left(\mathrm{F}_{\mathrm{V}} / \mathrm{F}_{\mathrm{M}}\right)$ relative to time zero. (b) Photochemical quenching, qL. C. nonphotochemical quenching, NPQ (n=3).

FIGURE 8. Production of reactive oxygen species in UWO 241 (P) vs. SAG 49.72 (M) during short-term incubation in low temperature $\left(5^{\circ} \mathrm{C}\right)$ or high light $\left(300 \mu \mathrm{mol} \mathrm{m} \mathrm{m}^{-2} \mathrm{~s}^{-1}\right)$ stress. Algal samples were incubated for 1 hour in the presence of NBT dye to detect superoxide. Data is normalized to time 0. Letters - a, statistical significance between control vs. stress within one algal species; b, statistical significance between UWO 241 vs. SAG 49.72 when grown under same treatment $(\mathrm{n}=3 ; \mathrm{p}<0.05)$.

Table 1. Growth conditions for UWO 241 and SAG 49.72 used in this study.

\begin{tabular}{lll}
\hline Growth Condition & UWO 241 & SAG 49.72 \\
\hline Control (C) & $8^{\circ} \mathrm{C} / 0.43 \mathrm{mM} \mathrm{NaCl}^{\circ} 50 \mu \mathrm{mol} \mathrm{m}^{-2} \mathrm{~s}^{-1}$ & $20^{\circ} \mathrm{C} / 0.43 \mathrm{mM} \mathrm{NaCl} / 50 \mu \mathrm{mol} \mathrm{m}^{-2} \mathrm{~s}^{-1}$ \\
High light (HL) & $250 \mu \mathrm{mol} \mathrm{m} \mathrm{s}^{-2} \mathrm{~s}^{-1}$ & $500 \mu \mathrm{mol} \mathrm{m} \mathrm{s}^{-2}$ \\
Low temperature (LT) & $2^{\circ} \mathrm{C}$ & $11^{\circ} \mathrm{C}$ \\
High Salinity (HS) & $700 \mathrm{mM} \mathrm{NaCl}$ & $100 \mathrm{mM} \mathrm{NaCl}$ \\
\hline
\end{tabular}




\begin{tabular}{|c|c|c|c|c|c|}
\hline $\begin{array}{l}\text { Growth } \\
\text { Condition }\end{array}$ & $\begin{array}{l}\text { Max Growth } \\
\text { Rate }\left(\mathrm{hr}^{-1}\right)\end{array}$ & $\mathrm{F}_{\mathrm{V}} / \mathrm{F}_{\mathrm{M}}$ & $\mathrm{qL}$ & $\begin{array}{l}\text { Total } \\
\text { Chlorophyll ( } \mu \mathrm{g} \\
\left.\mathrm{ml}^{-1}\right)\end{array}$ & $\mathrm{Chl} \mathrm{a/b}$ \\
\hline UWO 241 & UWO 241 & UWO 241 & & & \\
\hline $\mathrm{C}$ & $0.031 \pm 0.002$ & $0.67 \pm 0.02^{\mathrm{b}}$ & $0.72 \pm 0.08^{\mathrm{b}}$ & $6.99 \pm 1.09$ & $1.00 \pm 0.02^{\mathrm{b}}$ \\
\hline HL & $0.034 \pm 0.001$ & $0.60 \pm 0.01^{\mathrm{a}}$ & $0.60 \pm 0.05^{\mathrm{b}}$ & $4.02 \pm 0.45^{\mathrm{a}, \mathrm{b}}$ & $0.80 \pm 0.14^{\mathrm{b}}$ \\
\hline LT & $\begin{array}{l}0.018 \pm \\
0.002^{\mathrm{a}, \mathrm{b}}\end{array}$ & $0.55 \pm 0.02^{\mathrm{a}, \mathrm{b}}$ & $0.82 \pm 0.04^{\mathrm{b}}$ & $4.79 \pm 0.59^{\mathrm{a}}$ & $0.97 \pm 0.10^{\mathrm{b}}$ \\
\hline HS & $0.034 \pm 0.002^{\mathrm{b}}$ & $0.60 \pm 0.05^{\mathrm{b}}$ & $0.70 \pm 0.10^{\mathrm{b}}$ & $5.06 \pm 0.46$ & $0.90 \pm 0.10^{\mathrm{b}}$ \\
\hline SAG 49.72 & SAG 49.72 & SAG 49.72 & & & \\
\hline $\mathrm{C}$ & $0.030 \pm 0.001$ & $0.70 \pm 0.02$ & $0.94 \pm 0.04$ & $9.26 \pm 1.62$ & $2.28 \pm 0.77$ \\
\hline HL & $0.087 \pm 0.002^{\mathrm{a}}$ & $0.65 \pm 0.04$ & $0.24 \pm 0.00^{\mathrm{a}}$ & $6.86 \pm 0.08$ & $4.29 \pm 0.29$ \\
\hline LT & $0.024 \pm 0.001^{\mathrm{a}}$ & $0.66 \pm 0.01^{\mathrm{a}}$ & $1.00 \pm 0.00$ & $4.31 \pm 0.15^{\mathrm{a}}$ & $5.66 \pm 0.29^{\mathrm{a}}$ \\
\hline $\mathrm{HS}$ & $0.016 \pm 0.002^{\mathrm{a}}$ & $0.66 \pm 0.04$ & $0.94 \pm 0.09$ & $8.13 \pm 0.84$ & $3.51 \pm 0.05$ \\
\hline
\end{tabular}

Table 2. Growth physiology parameters in cultures of UWO 241 and SAG 49.72 grown under control versus long-term stress conditions. Values are means with standard deviations ( $\mathrm{n}=3$ biological replicates). Superscript letters - ${ }^{\mathrm{a}}$, statistical significance between control vs. stress within one algal species; ${ }^{\mathrm{b}}$, statistical significance between UWO 241 vs. SAG 49.72 when grown under same treatment $(\mathrm{p}<0.05) . \mathrm{F}_{\mathrm{V}} / \mathrm{F}_{\mathrm{M}}$, maximum photochemical efficiency; qL, photochemical quenching.

Table 3. Gaussian fitting parameters for the subband decomposition of $77 \mathrm{~K} \mathrm{Chl} \mathrm{a} \mathrm{fluorescence} \mathrm{spectra}$ of SAG 49.72 and UWO 241 acclimated to control versus long-term stress conditions. $\lambda_{M A \Xi}$, maximum fluorescence emission peak (nm); Area \%, percentage area of individual bands calculated from total area of all bands; FWHM, full width at half-maximum. PSII/PSI was calculated as the ratio of the areas of peaks $\lambda 1$ and $\lambda 4$.

\begin{tabular}{lllll}
\hline SAG 49.72 & C & HL & LT & HS \\
\hline $1 \lambda_{M A \Xi}$ & 684.1 & 683.1 & 684.8 & 684.0 \\
FWHM & 12.52 & 11.51 & 12.96 & 12.21 \\
Area \% & 30.95 & 28.12 & 20.55 & 22.18 \\
$2 \lambda_{M A \Xi}$ & 696.4 & 693.5 & 695.0 & 695.1 \\
FWHM & 10.72 & 12.31 & 2.87 & 2.01 \\
Area \% & 7.90 & 5.46 & 0.23 & 0.25 \\
$3 \lambda_{M A \Xi}$ & 704.1 & 700.7 & 698.4 & 697.9 \\
FWHM & 8.91 & 8.20 & 11.69 & 10.17 \\
Area \% & 1.13 & 1.34 & 7.86 & 3.30 \\
$4 \lambda_{M A \Xi}$ & 713.1 & 713.5 & 714.9 & 711.0 \\
FWHM & 25.08 & 26.46 & 27.38 & 31.62 \\
Area \% & 24.85 & 33.57 & 47.18 & 57.60 \\
$5 \lambda_{M A \Xi}$ & 724.5 & 724.6 & 734.6 & 741.2 \\
FWHM & 58.68 & 53.07 & 44.47 & 16.66 \\
Area \% & 35.17 & 31.50 & 24.18 & 43.91 \\
PSII/PSI & $\mathbf{1 . 2 4}$ & $\mathbf{0 . 8 4}$ & $\mathbf{0 . 4 3}$ & $\mathbf{0 . 3 8}$ \\
UWO 241 & $\mathbf{C}$ & $\mathbf{H L}$ & $\mathbf{L T}$ & $\mathbf{H S}$ \\
$1 \lambda_{M A \Xi}$ & 683.5 & 683.1 & 683.2 & 682.7 \\
FWHM & 12.11 & 11.81 & 11.98 & 11.46 \\
Area \% & 35.81 & 36.15 & 43.14 & 47.10 \\
$2 \lambda_{M A \Xi}$ & 695.7 & 695.4 & 695.6 & 695.3 \\
FWHM & 5.82 & 14.68 & 6.91 & 13.80
\end{tabular}




\begin{tabular}{lllll}
\hline SAG 49.72 & C & HL & LT & HS \\
\hline Area \% & 0.90 & 14.92 & 1.78 & 14.99 \\
$3 \lambda_{M A \Xi}$ & 697.1 & - & 696.8 & - \\
FWHM & 15.24 & - & 16.40 & - \\
Area \% & 18.02 & - & 19.25 & - \\
$4 \lambda_{M A \Xi}$ & 710.4 & 710.4 & 711.4 & 705.9 \\
FWHM & 24.93 & 23.58 & 23.70 & 38.24 \\
Area \% & 24.51 & 15.27 & 16.63 & 35.92 \\
$5 \lambda_{M A \Xi}$ & 734.1 & 722.3 & 735.1 & 742.2 \\
FWHM & 42.59 & 71.47 & 48.34 & 8.75 \\
Area \% & 20.76 & 33.40 & 19.20 & 1.12 \\
PSII/PSI & $\mathbf{1 . 4 6}$ & $\mathbf{1 . 5 3}$ & $\mathbf{1 . 8 2}$ & $\mathbf{1 . 2 3}$ \\
\hline
\end{tabular}
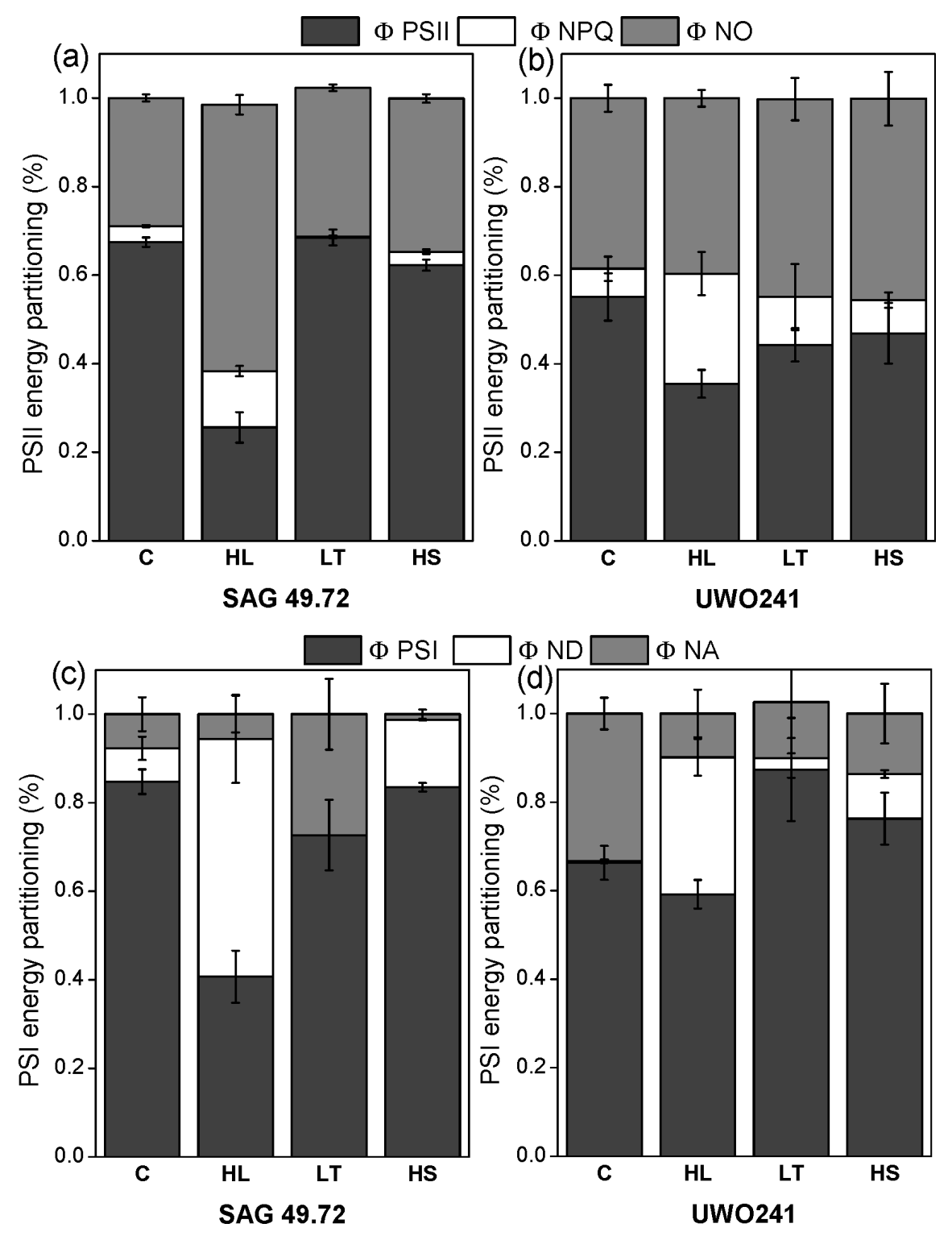

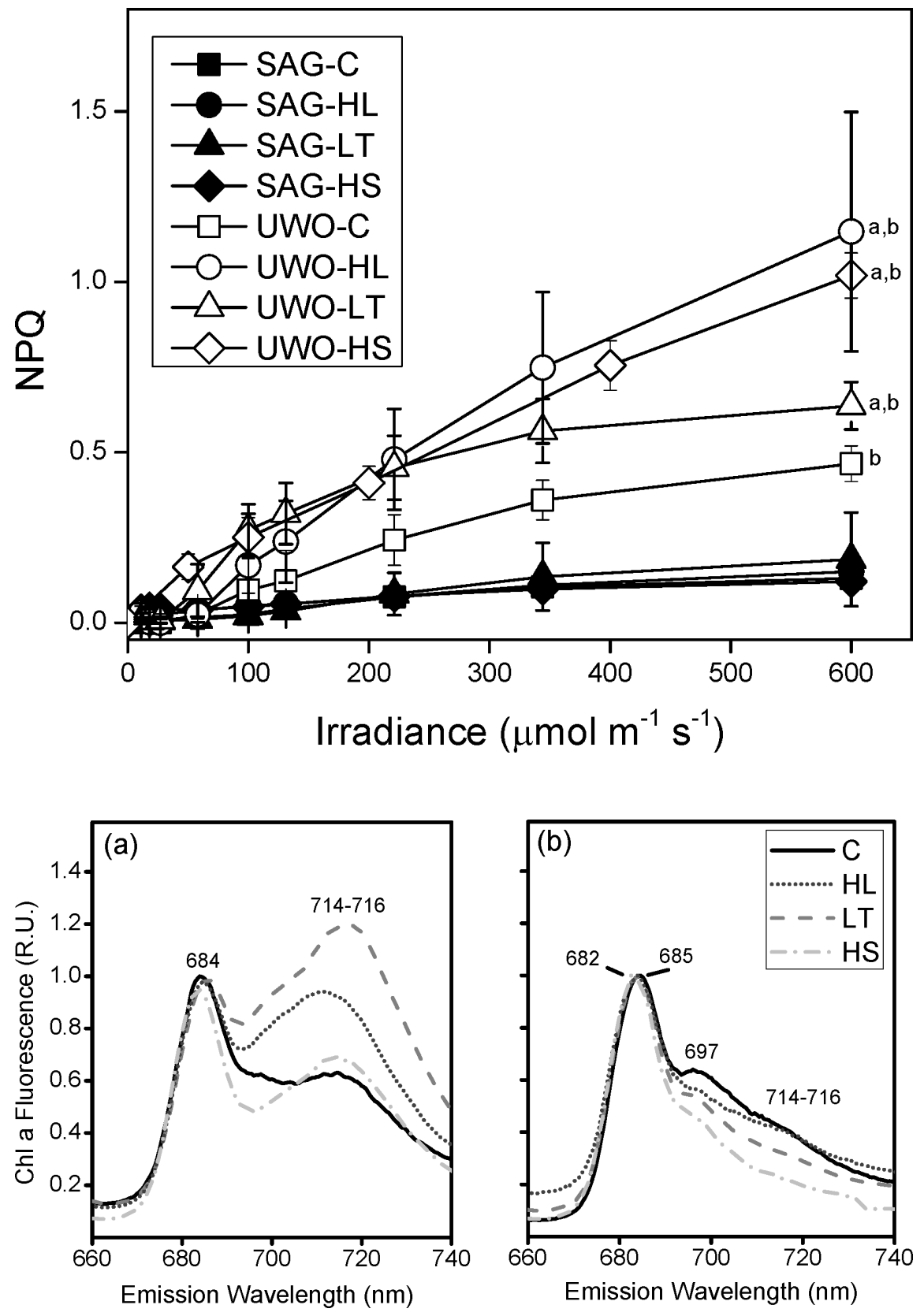

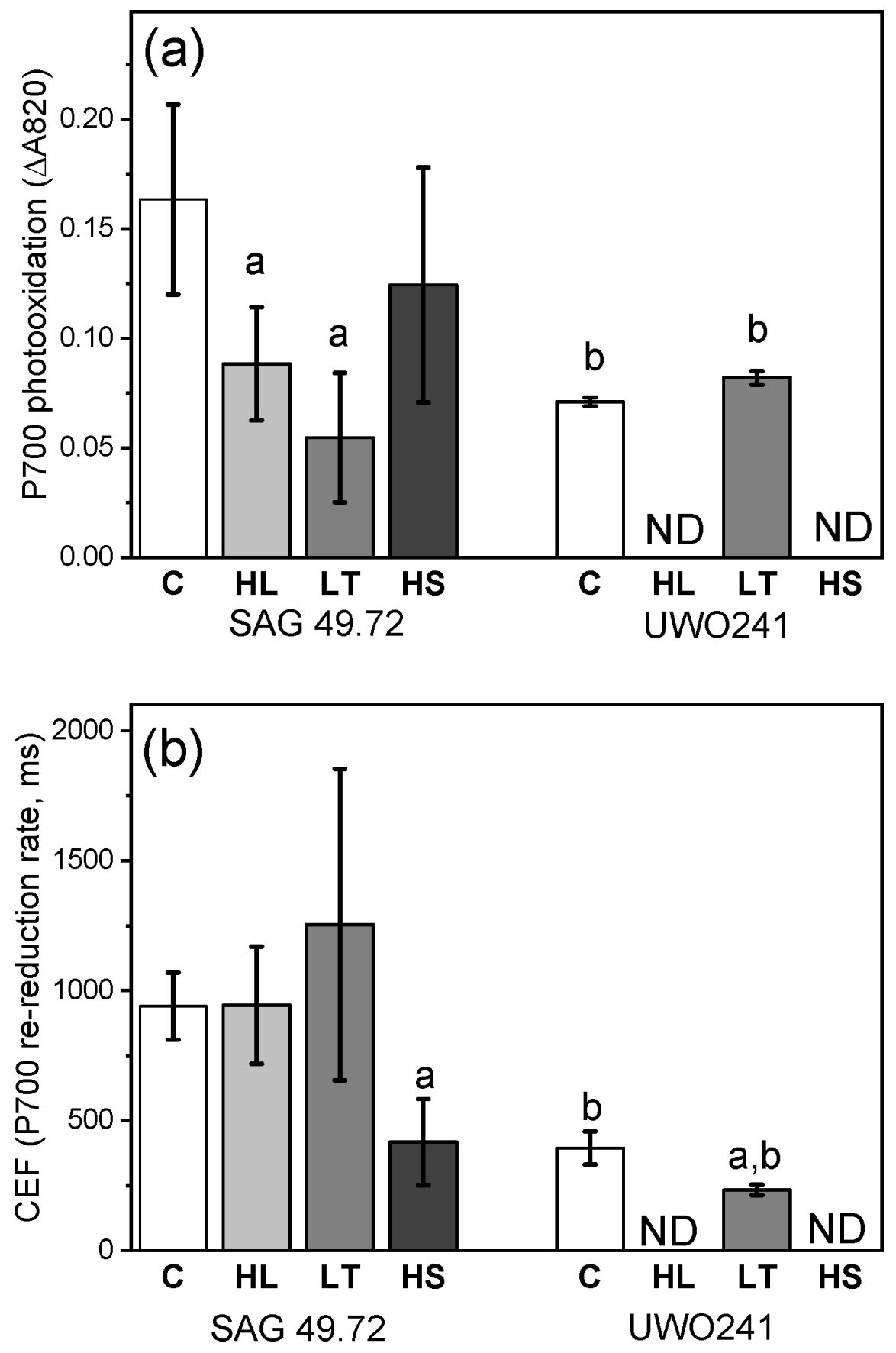

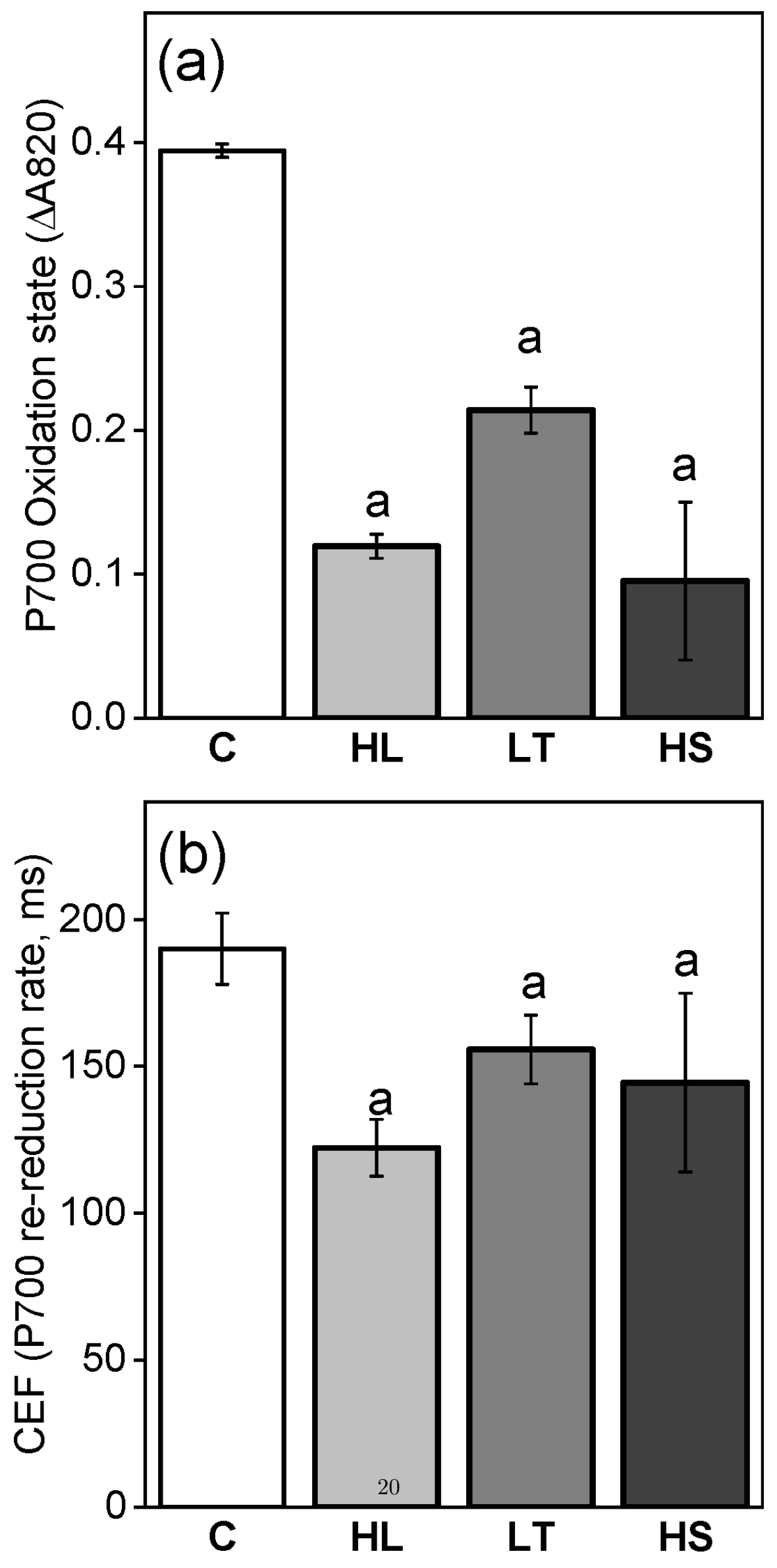

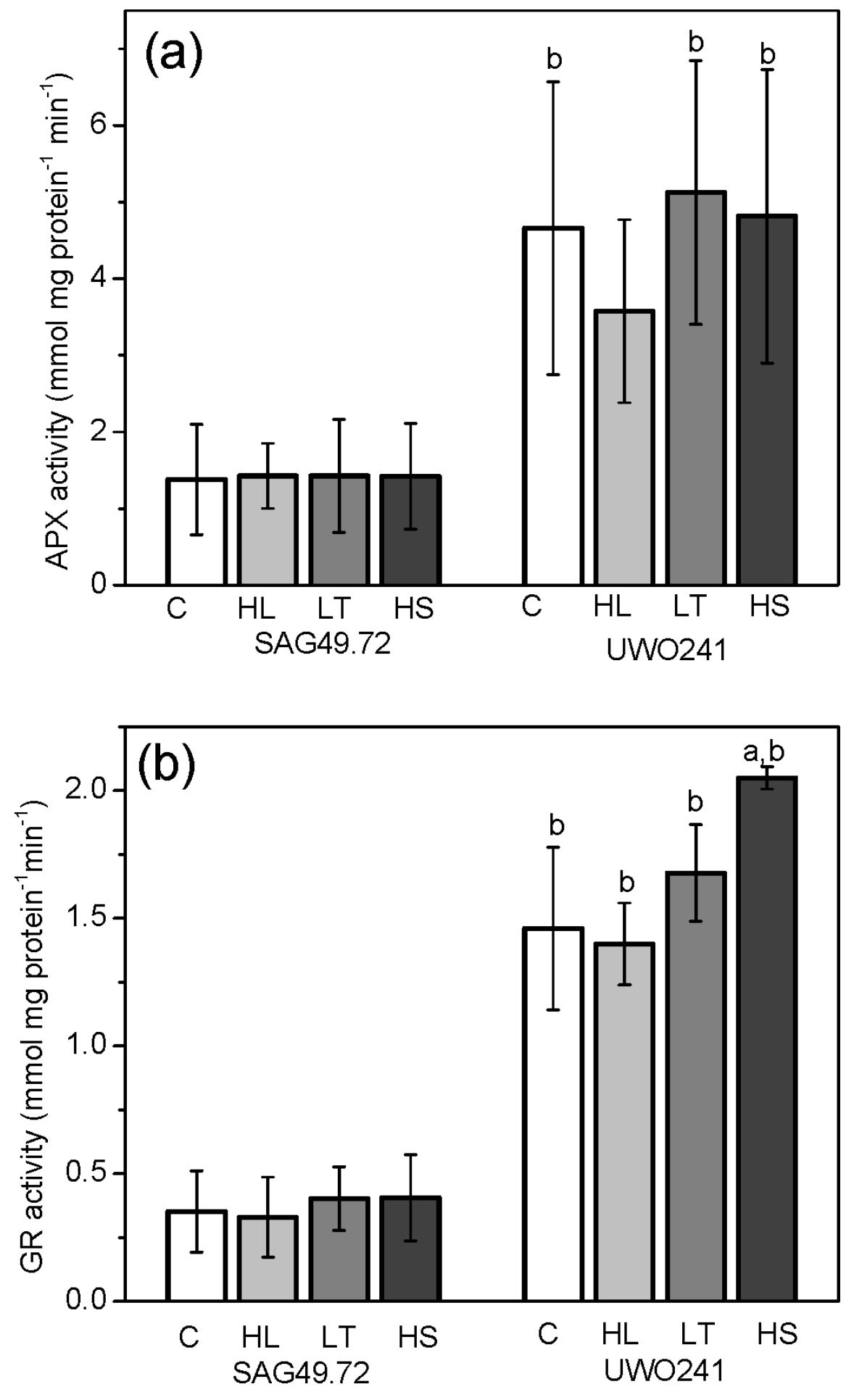

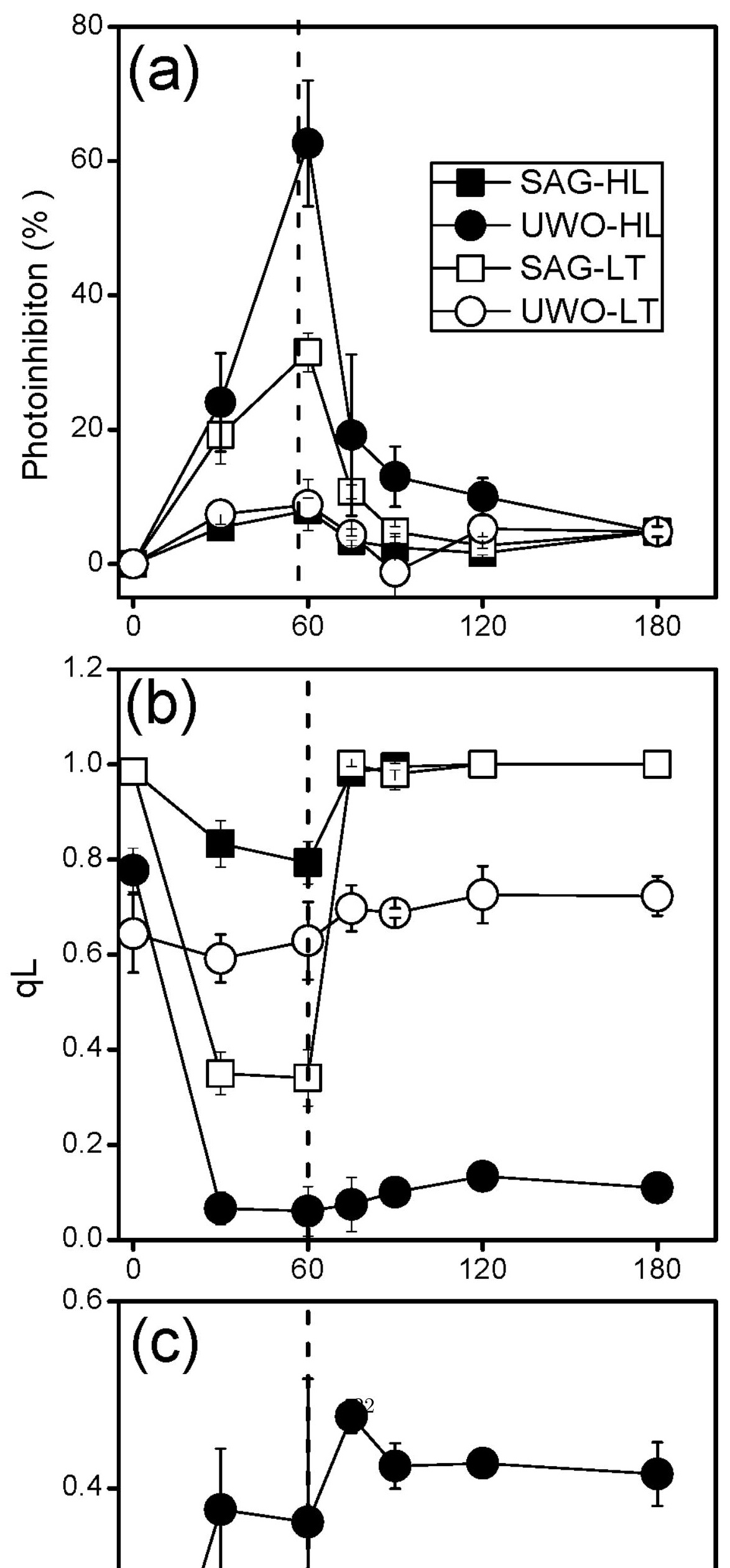

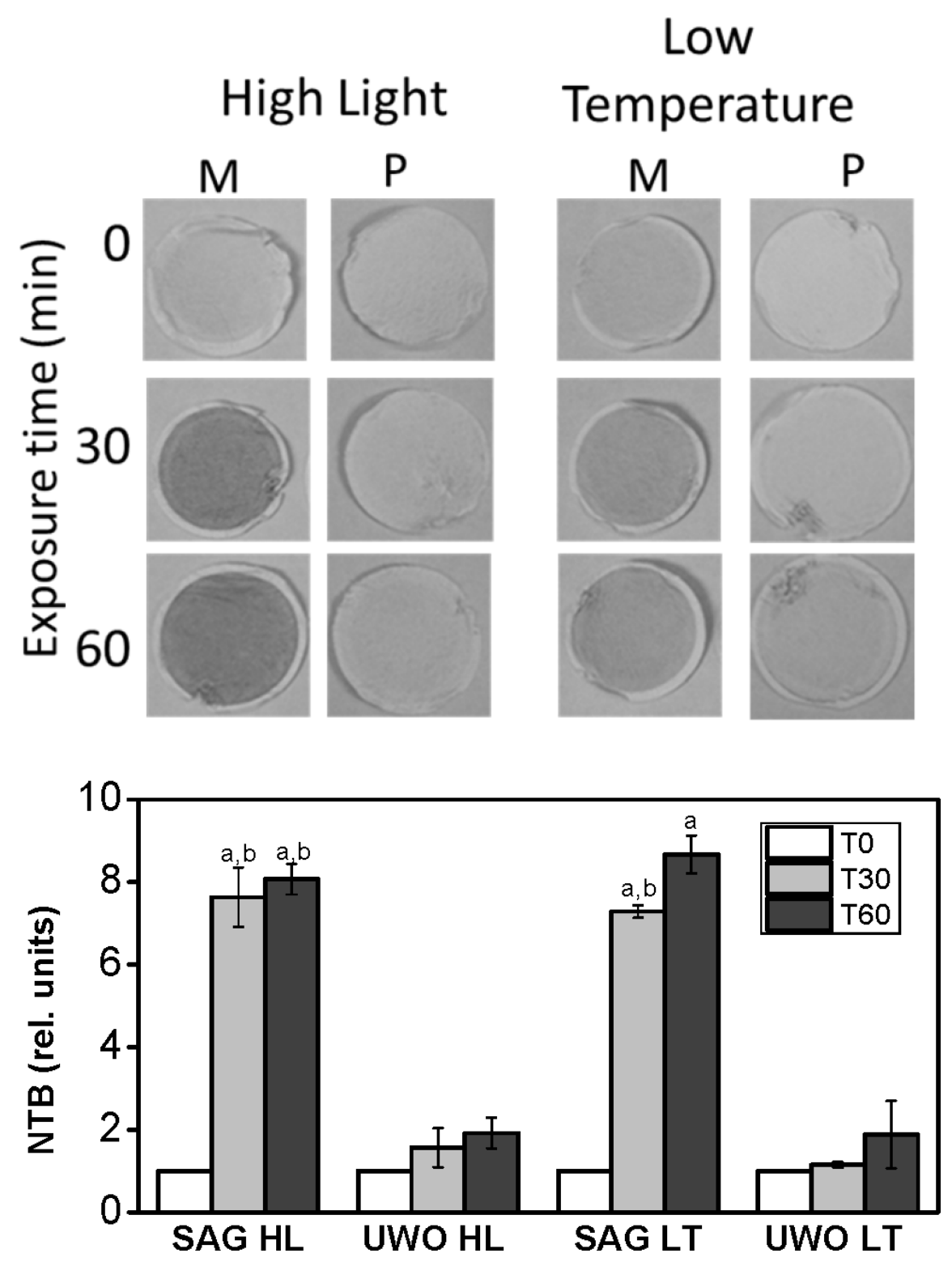